\title{
Circular RNA hsa_circ_101555 promotes hepatocellular carcinoma cell proliferation and migration by sponging miR-145-5 $p$ and regulating CDCA3 expression
}

\author{
Xiaoguang Gu, ${ }^{1,2}$, Jianan Zhang ${ }^{1,2}$, Yajuan Ran ${ }^{2,3}$, Hena Pan ${ }^{1,2}$, JinHong Jia ${ }^{1,2}$, Ying Zhao ${ }^{1,2}$, Xijuan Zhao ${ }^{1,2}$, Wendi Li, ${ }^{2,4}$,
} Shasha Song ${ }^{5}$ and Xiufeng $\mathrm{Yu}^{1,2,3}$

\begin{abstract}
Circular RNAs have been reported to play significant roles in regulating pathophysiological processes while also guiding clinical diagnosis and treatment of hepatocellular carcinoma (HCC). However, only a few circRNAs have been identified thus far. Herein, we investigated the role of a specific closed-loop structure of hsa_circ_101555 that was generated by back-splicing of the host gene casein kinase 1 gamma 1 (CSNK1G1) in the development and proliferation of HCC. We investigated the expression of Hsa_circ_101555 in HCC and normal tissues using bioinformatics. The expression level of hsa_circ_101555 was further detected by fluorescence in situ hybridization and qRT-PCR in ten HCC patients. Transwell, migration, WST-1 assays, and colony formation assays were used to evaluate the role of hsa_circ_101555 in HCC development and proliferation. The regulatory mechanisms of hsa_circ_101555 in miR-145-5p and CDCA3 were determined by dual luciferase reporter assay. A mouse xenograft model was also used to determine the effect of hsa_circ_101555 on HCC growth in vivo. hsa_circ_101555 showed greater stability than the linear RNA; while in vitro and in vivo results demonstrated that hsa_circ_101555 silencing significantly suppressed cell proliferation, migration, and invasion of HCC cells. Rescue experiments further demonstrated that suppression of miR145-5p significantly attenuated the biological effects of hsa_circ_101555 knockdown in HCC cells. We also identified a putative oncogene CDCA3 as a potential miR-145-5p target. Thus, our results demonstrated that hsa_circ_101555 might function as a competing endogenous RNA of miR-145-5p to upregulate CDCA3 expression in HCC. These findings suggest that hsa_circ_101555 may be a potential therapeutic target for patients with HCC.
\end{abstract}

\section{Introduction}

Hepatocellular carcinoma (HCC), the most prevalent form of primary liver cancer, represents one of the most common malignant tumors globally ${ }^{1}$. HCC is an aggressive disease with dismal prognosis, constituting the third

\footnotetext{
Correspondence: Xiufeng Yu (yuxiufeng0806@163.com)

${ }^{1}$ College of Medical Laboratory Science and Technology, Harbin Medical University (Daqing), 163319 Daqing, P. R. China

${ }^{2}$ Central Laboratory of Harbin Medical University (Daqing), 163319 Daqing, P. R. China

Full list of author information is available at the end of the article

These authors contributed equally: Xiaoguang Gu, Jianan Zhang, Yajuang Ran Edited by G. Blandino
}

leading cause of cancer-related deaths worldwide ${ }^{2}$. Despite advances in the clinical understanding of the underlying mechanisms in $\mathrm{HCC}$ development and progression, its 5 -year survival rate remains low $^{3}$. Additionally, the molecular pathogenesis and therapeutic targets in HCC remain largely unknown. Therefore, understanding the pathogenic process of $\mathrm{HCC}$ and its regulatory mechanisms would significantly aid its management.

Circular RNAs (circRNAs) are a newly discovered noncoding RNA (ncRNA) that ubiquitously exist in several species ${ }^{4,5}$. Unlike canonical linear RNAs, circRNAs form a covalently closed continuous loop structure that

\section{(c) The Author(s) 2021}

(c) (i) Open Access This article is licensed under a Creative Commons Attribution 4.0 International License, which permits use, sharing, adaptation, distribution and reproduction c. in any medium or format, as long as you give appropriate credit to the original author(s) and the source, provide a link to the Creative Commons license, and indicate if changes were made. The images or other third party material in this article are included in the article's Creative Commons license, unless indicated otherwise in a credit line to the material. If material is not included in the article's Creative Commons license and your intended use is not permitted by statutory regulation or exceeds the permitted use, you will need to obtain permission directly from the copyright holder. To view a copy of this license, visit http://creativecommons.org/licenses/by/4.0/. 
lack $5^{\prime}$ caps and $3^{\prime}$ polyadenylated tails, making them more stable than linear RNAs ${ }^{6}$. circRNAs reportedly function as molecular sponges for microRNAs (miRNAs) ${ }^{7,8}$ and RNA-binding proteins ${ }^{9}$, while also serving as vital regulators of gene transcription and expression ${ }^{10,11}$. Furthermore, circRNAs are highly conserved across multiple species and exhibit tissue-specific and development stage-dependent expression patterns ${ }^{12,13}$. These features imply that circRNAs possess significant functions in biological and pathological processes.

Recent studies have reported that circRNAs are differentially expressed in HCC and serve a central role in its carcinogenesis and progression ${ }^{14-20}$. Thus, circRNAs may represent promising diagnostic markers and therapeutic targets for HCC. However, compared with other ncRNAs, such as miRNAs and long noncoding RNAs, the research on circRNA in HCC remains in its infancy. To date, only a few functional circRNAs have been discovered and characterized in $\mathrm{HCC}^{21}$, while a large number remain to be explored or identified.

In the present study, we analyzed the expression profiles of three circRNA in human HCC tissues and identified hsa_circ_101555 to be conserved and significantly upregulated. Therefore, we focused on investigating the role of hsa_circ_101555 in the development and proliferation of $\mathrm{HCC}$ with respect to the miR-145-5p/CDCA3 signaling axis.

\section{Results}

hsa_circ_101555 is upregulated in HCC

To identify unique circRNAs involved in HCC, we analyzed the microarray data of GSE7852, GSE94508, and GSE97322 datasets downloaded from the Gene Expression Omnibus (GEO) database. We then visualized the differentially expressed circRNAs (DEcircRNAs) in HCC and normal tissue samples using the "limma" package of $\mathrm{R}$ software. A false discovery rate $<0.05$ and $\mid \log 2$ foldchange $>1$ were set as the cutoff criteria for screening the DEcircRNAs (Fig. 1A-C). Among the top 25 upregulated DEcircRNAs, only hsa_circ_101555 appeared in all three GSE datasets (Fig. 1D). Thus, we focused on investigating its role in HCC progression. Subsequently, according to circBase (http://www.circbase.org), we found that hsa_circ101555 was derived from the host gene casein kinase 1 gamma 1 (CSNK1G1), consisting of six exons (exon 1-6, $815 \mathrm{nt})$ cyclized by the head-to-tail splicing of exon 1 and exon 6. The existence of a back-spliced junction was confirmed by Sanger sequencing (Fig. 1E). We further verified the expression of CSNK1G1 in HCC tissues, and found it to be upregulated (Supplementary Fig. 1A). Moreover, analysis of the overall survival and CSNK1G1 expression using GEPIA (http://gepia.cancer-pku.cn/) revealed that high CSNK1G1 expression in HCC was not associated with overall survival (Supplementary Fig. 1B-C).

\section{hsa_circ_101555 is primarily localized in HCC cell cytoplasm}

In general, the subcellular localization of circRNA determines its primary mode of action. Fluorescent in situ hybridization (FISH) analysis revealed that hsa_circ_101555 expression was higher in tumor tissues than in matched nontumor sections (Fig. 2A). Furthermore, qRT-PCR examination confirmed that hsa_circ_101555 expression was markedly higher in HCC tissues than in adjacent normal tissues (Fig. 2B). High hsa_circ_101555 expression in $\mathrm{HCC}$ was associated with overall survival (Supplementary Fig. 2A).

We also analyzed hsa_circ_101555 expression within the serum of HCC patients and healthy controls and found the expression to be significantly higher in $\mathrm{HCC}$ patients compared to healthy controls (Supplementary Fig. 3A). Meanwhile, we also observed that hsa_circ_101555 was upregulated in III-IV stage HCC compared to I-II stage patients, based on TNM stage (Supplementary Fig. 3B). Additionally, the expression of hsa_circ_101555 was higher in all HCC cell lines (HepG2, SK-Hep-1, Huh-7, and HCCLM3) compared to normal liver LO2 cells, with a significantly higher expression in HepG2 and HCCLM3 cells than in Huh-7 and SK-Hep-1 cells (Fig. 2C).

The circular structure of hsa_circ_101555 was confirmed using RNase R. As shown in Fig. 2D, amplified linear transcripts of CSNK1G1 were clearly degraded by RNase R, while hsa_circ_101555 was resistant to degradation. These data demonstrated both the presence and circular structure of hsa_circ_101555 in HCC cells (HepG2 and HCCLM3). Moreover, hsa_circ_101555 was predominantly located in the cytoplasm (Fig. 2E-F).

\section{hsa_circ_101555 acts as an oncogene in HCC}

To further investigate the regulatory role of hsa_circ 101555, we designed three hsa_circ_101555 small interfering RNAs (siRNAs) to specifically target different binding sites on the back-splice junction sequence of hsa_circ_101555. As si-hsa_circ_101555_001 and si-hsa_circ_101555_003 effectively silenced hsa_circ_101555 expression in HCCLM3 and HepG2 cell lines, they were used for subsequent experiments (Fig. 2G). We also generated stable hsa_circ_101555 overexpressing Huh-7 and SK-Hep-1 cells through lentiviral transduction and observed that lv-hsa_circ_101555 treatment caused marked upregulation of hsa_circ_101555 in these cells (Fig. 2H).

Subsequently, we detected the effect of hsa_circ_101555 knockdown and overexpression on HCC tumor progression in vitro. WST-1 assay results showed that hsa_circ_101555 silencing reduced HCCLM3 and HepG2 cell proliferation (Fig. 3A and B), whereas its overexpression had the opposite effect in these cells (Fig. 3C and D). Colony formation assays further revealed that hsa_circ_101555 was positively associated with HCCLM3 


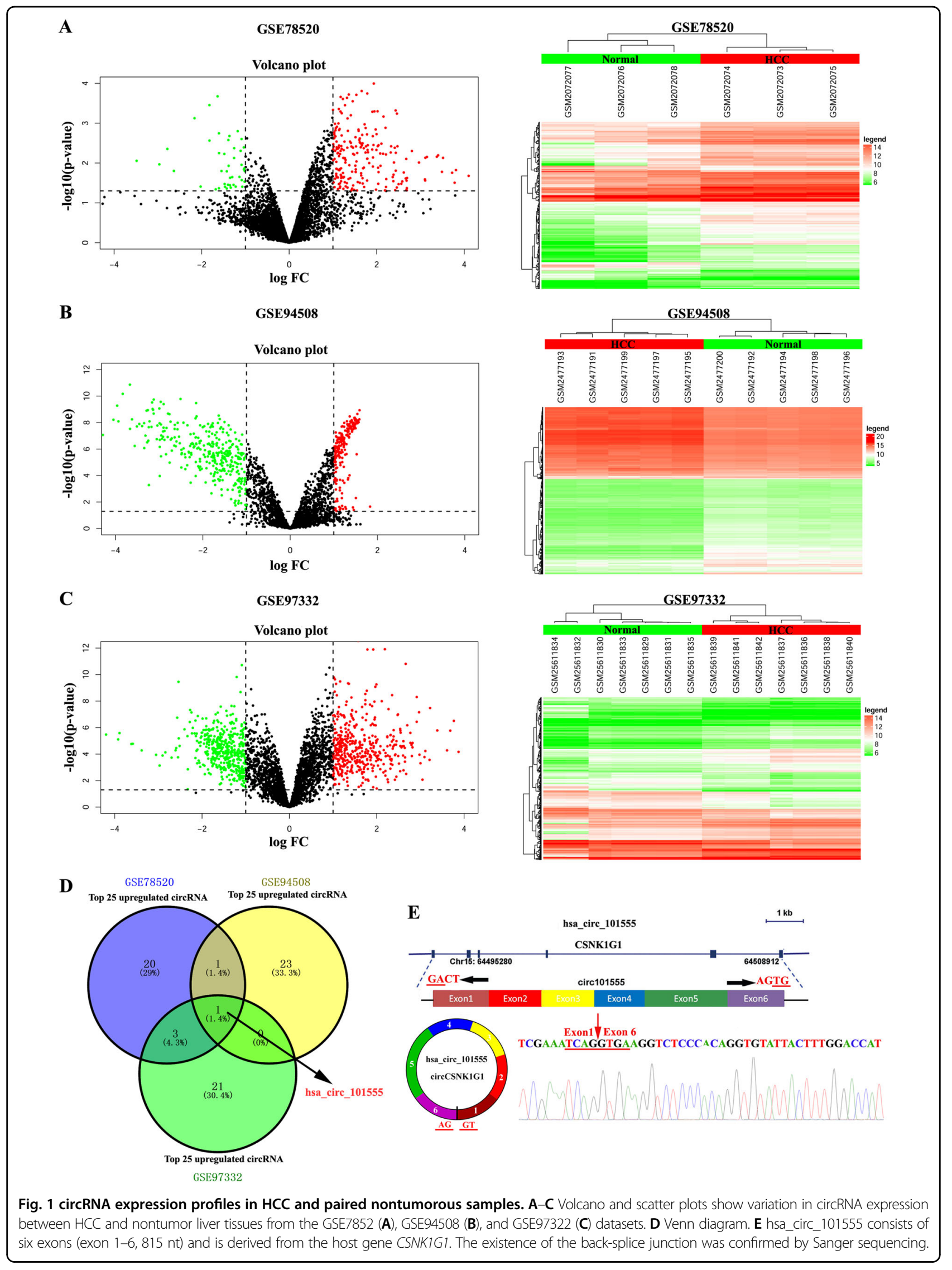




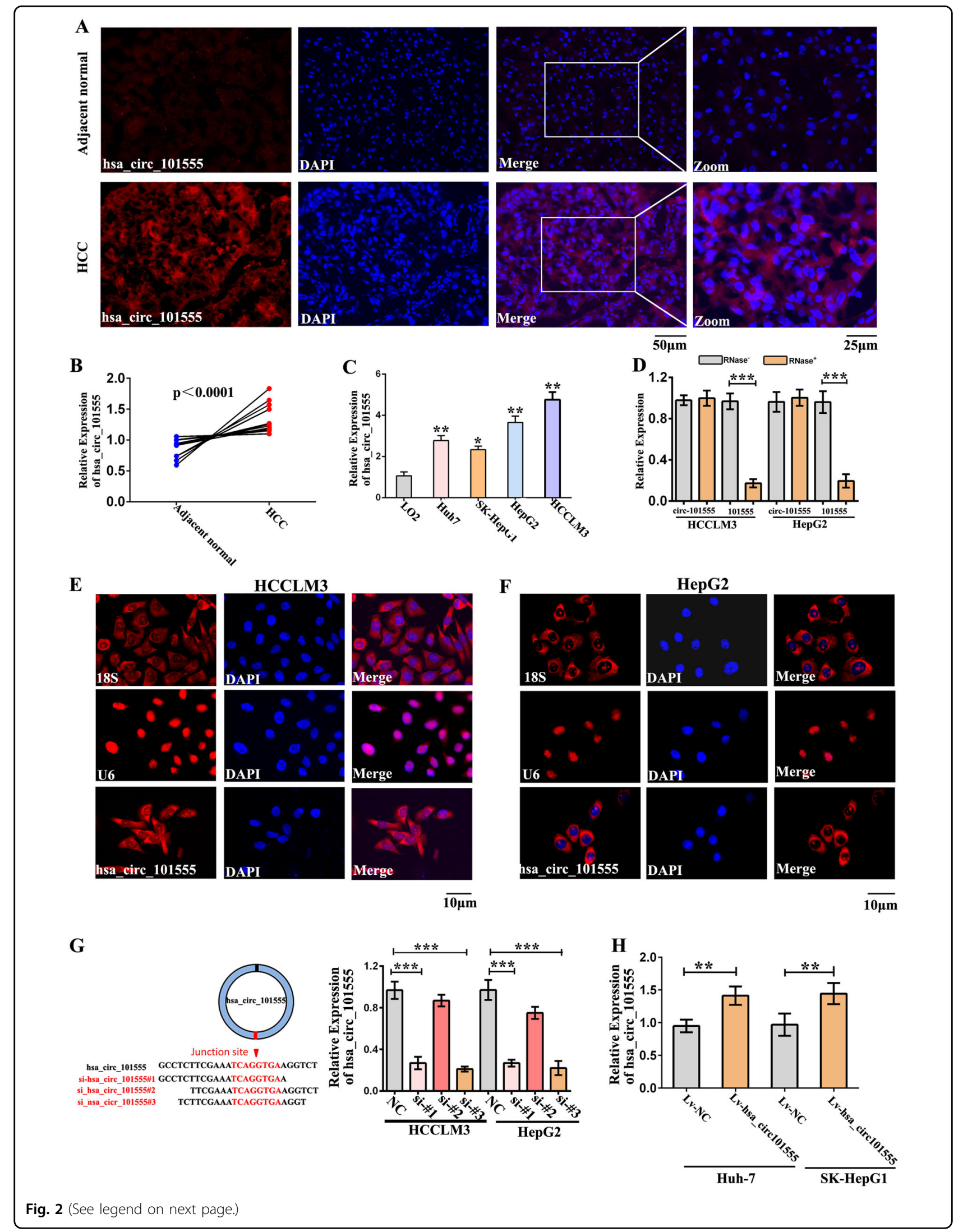




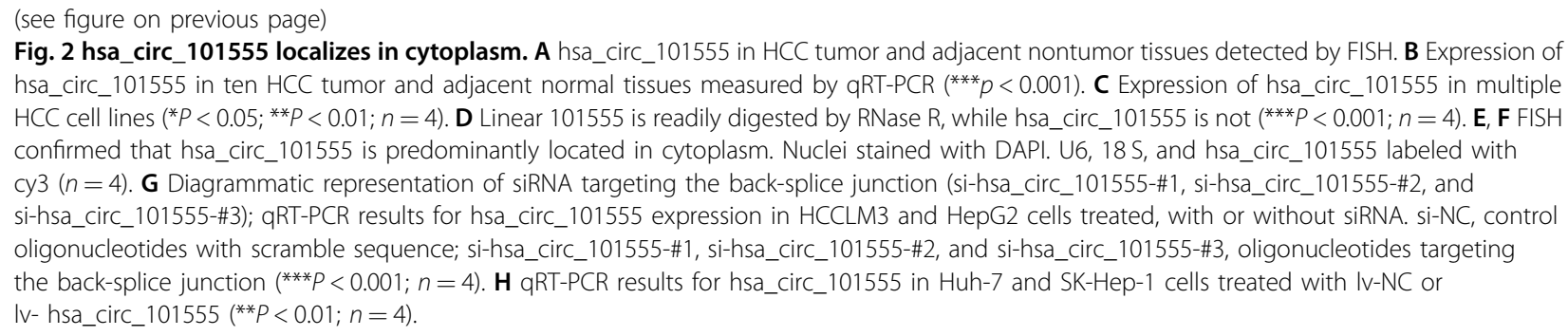

and HepG2 cell proliferation (Fig. 3E), whereas hsa_circ_101555 overexpression had the opposite effect on Huh-7 and SK-Hep-1 cells (Fig. 3F). Notably, proliferating cell nuclear antigen levels in these cells were initially elevated, however, became attenuated following transfection with the hsa_circ_101555 back-splice junctionspecific siRNA (Supplementary Fig. 4A-B).

HCCLM3 and HepG2 cell migration and invasion were also suppressed by hsa_circ_101555 silencing (Fig. 3G and I), whereas these properties were enhanced by hsa_circ_101555 overexpression in Huh-7 and SK-Hep1 cells (Fig. $3 \mathrm{H}$ and J). We further evaluated the effect that hsa_circ_101555 has on the expression of migration markers in HCC cell lines. Western blot analysis revealed that hsa_circ_101555 silencing inhibited the expression of fibronectin and vimentin, while that of Ecadherin was increased in HCCLM3 and HepG2 cells (Supplementary Fig. 5A-B). Moreover, Hsa_circ_101555 overexpression significantly decreased the relative expression of E-cadherin, while increasing that of vimentin and fibronectin in both Huh-7 and SK-HepG1 cells (Supplementary Fig. 5C-D). Taken together, these results suggest that hsa_circ_101555 was required to sustain the proliferation, migration, and invasion of $\mathrm{HCC}$ cells in vitro.

\section{The RNA-binding protein EIF4A3 regulates hsa_circ_101555 expression}

EIF4A3, a core component of the exon junction complex, has been shown to play an essential role in pre-mRNA splicing ${ }^{22}$, while also inducing circRNA expression via binding to the upstream or downstream regions of host gene mRNA and inducing circular RNA formation ${ }^{23-25}$. In fact, we discovered four putative binding sites for EIF4A3 in the upstream and downstream region of the CSNK1G1 mRNA transcript (CSNK1G1 pre-mRNA) via CircInteractome (https:// circinteractome.nia.nih.gov/index.html) (Fig. 4A).

We conduced pull-down assay using CSNK1G1 mRNA and confirmed the enrichment of EIF4A3 protein in the pull-down of CSNK1G1 mRNA rather than control (Fig. 4B). The data from an RIP (RNA-binding protein immunoprecipitation) assay using anti-eIF4A3 antibody further indicated that eIF4A3 can bind CSNK1G1 mRNA through these four putative binding sites, which we designated as, a, b, c, and d, however, did not bind hsa_circ_101555 (designated e) in the corresponding RNA-protein complex (Fig. 4C). We then knocked down eIF4A3 and observed reduced hsa_circ_101555 expression and increased CSNK1G1 expression (Fig. 4C and Supplementary Fig. 6A). Meanwhile, overexpression of eIF4A3 increased hsa_circ_101555 and decreased host gene CSNK1G1 expression (Fig. 4D and Supplementary Fig. 6B). In contrast, silencing hsa_circ_101555 did not affect EIF4A3 expression (Fig. 4E). Taken together, EIF4A3 was observed to increase hsa_circ_101555 expression, potentially by combining with the flanking sequences.

\section{Identification of miRNAs that binding hsa_circ_101555}

Given that circRNAs may act as competing endogenous RNAs (ceRNA) for miRNAs and regulate mRNA expression, we assessed the potential hsa_circ_101555 targets via a ceRNA-dependent mechanism. First, we determined the expression profiles of miRNAs from GSE115016 and GSE4187 datasets in HCC and normal tissue samples using miRNA microarray (Fig. 5A). Based on the target prediction tool, miRNAs were found to contain binding sites for hsa_circ_101555. Subsequent Venn analysis indicated the possible involvement of miR145-5p in HCC (Fig. 5B). Therefore, we analyzed its expression in ten paired HCC and adjacent normal tissues by qRT-PCR and found it to be significantly downregulated in HCC tissues. Moreover, patients with low miR-145-5p level had a poorer 10-year overall survival (OV), compared to those with high miR-145-5p levels (Fig. $5 \mathrm{C}$ and D). Pearson correlation analysis further revealed a negative correlation $(p<0.0001 \mathrm{r}=-0.7364)$ between hsa_circ_101555 and miR-145-5p expression levels (Fig. 5E). Additionally, luciferase reporter assays using either a wild-type hsa_circ_101555 sequence or a mutated miR-145-5p binding sites, inserted into the $3^{\prime}$ untranslated region of Renilla luciferase, showed that miR-145-5p overexpression significantly reduced the luciferase activity of the wild-type reporter without impacting that the mutant sequence-bound reporter 


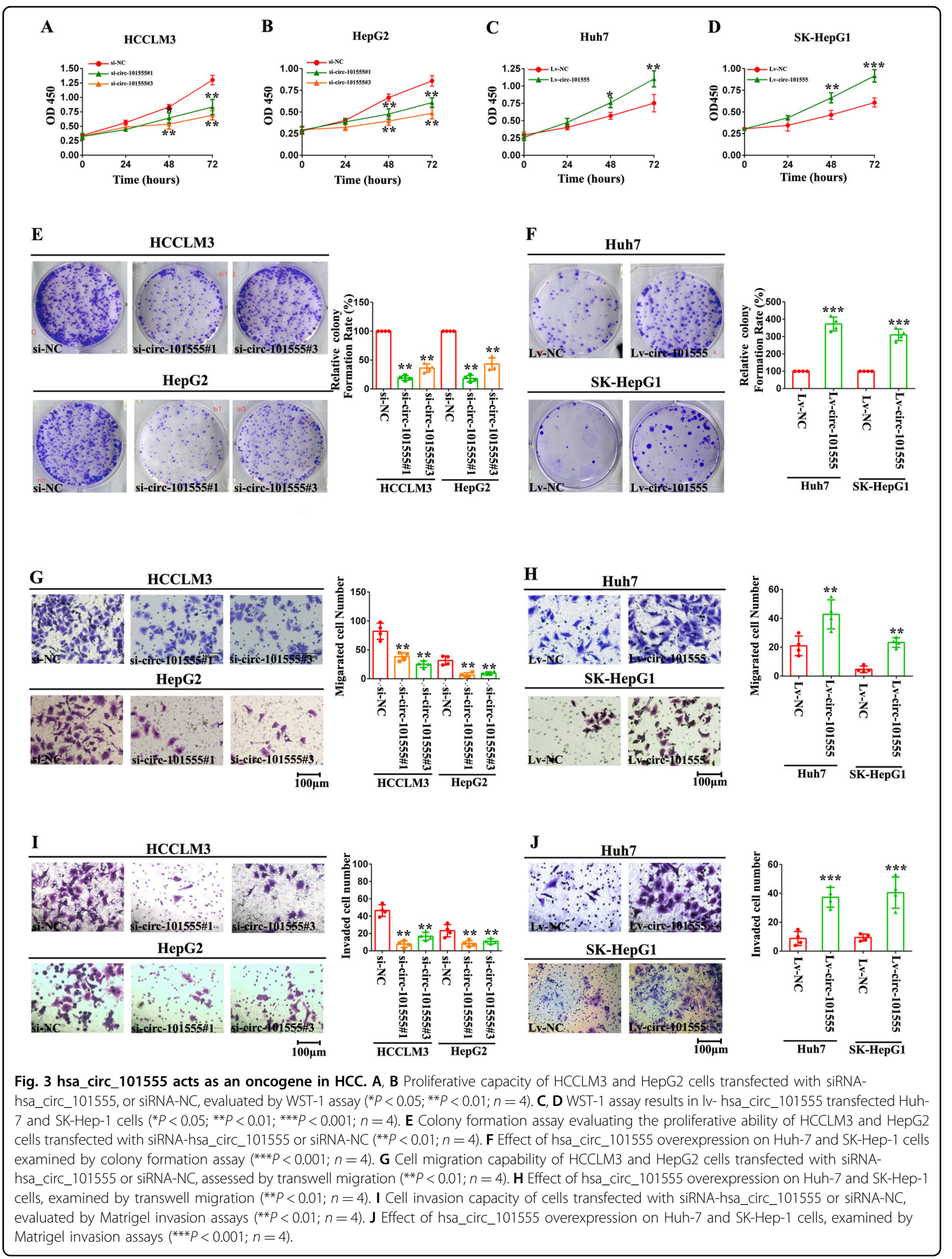


A

\begin{tabular}{|c|c|c|c|c|c|c|c|c|c|c|}
\hline circRNAs & Tag Name & \%identity & $\begin{array}{l}\text { Alignment } \\
\text { Length }\end{array}$ & $\begin{array}{l}\text { Mismatch } \\
\text { es }\end{array}$ & \begin{tabular}{|l} 
Cap \\
Openin \\
gs \\
\end{tabular} & $\begin{array}{l}\text { Tag } \\
\text { Start }\end{array}$ & $\begin{array}{l}\text { Tag } \\
\text { end }\end{array}$ & \begin{tabular}{|l} 
Circular \\
RNA \\
Star \\
\end{tabular} & $\begin{array}{l}\text { Circular } \\
\text { RNA } \\
\text { end }\end{array}$ & $\begin{array}{l}\text { Upstream/ } \\
\text { downstream }\end{array}$ \\
\hline Has_circ_0001955 & HHLE1_122387_eIF4AIII_rep1_122387_1_40 & $100 \%$ & 40 & 0 & 0 & 1 & 40 & -3 & 37 & upstream \\
\hline Has_circ_0001955 & HHLE1_295443_eIF4AIII_rep1_295443_1_28 & $100 \%$ & 28 & $\mathbf{0}$ & $\mathbf{0}$ & 1 & 28 & +372 & +399 & Downstream \\
\hline Has_circ_0001955 & HHLE1_412409_eIF4AIII_rep1_412409_1_37 & $100 \%$ & 37 & o & o & 1 & 37 & +953 & +989 & Downstream \\
\hline Has_circ_0001955 & HHLE1_412410_eIF4AIII_rep1_412410_1_39 & $100 \%$ & 39 & 0 & $\mathbf{0}$ & 1 & 39 & +882 & +920 & Downstream \\
\hline Has_circ_0001955 & HHLE1_412411_eIF4AIII_rep1_412411_1_29 & $100 \%$ & 29 & 0 & o & 1 & 29 & +602 & +630 & Downstream \\
\hline Has_circ_0001955 & HHLE1_412412_eIF4AIII_rep1_412412_1_52 & $100 \%$ & 52 & 0 & o & 1 & 52 & +547 & +598 & Downstream \\
\hline Has_circ_0001955 & HHLE1_412413_eIF4AIII_rep1_412413_1_25 & $100 \%$ & 25 & 0 & 0 & 140 & 25 & +142 & +166 & Downstream \\
\hline Has_circ_0001955 & HHLE1_412434_eIF4AIII_rep1_412434_1_44 & $100 \%$ & 44 & 0 & 0 & 1 & 44 & -566 & -523 & upstream \\
\hline
\end{tabular}

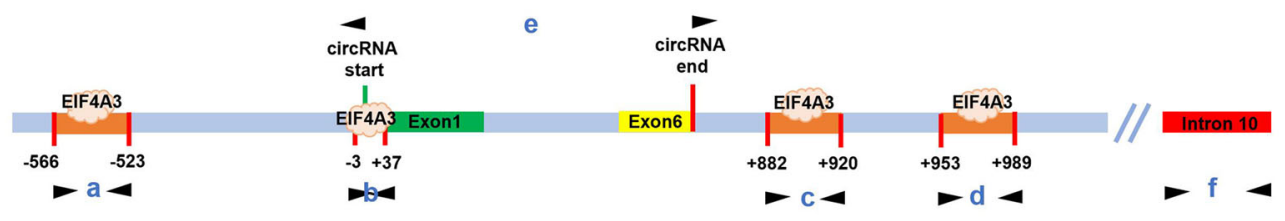

B

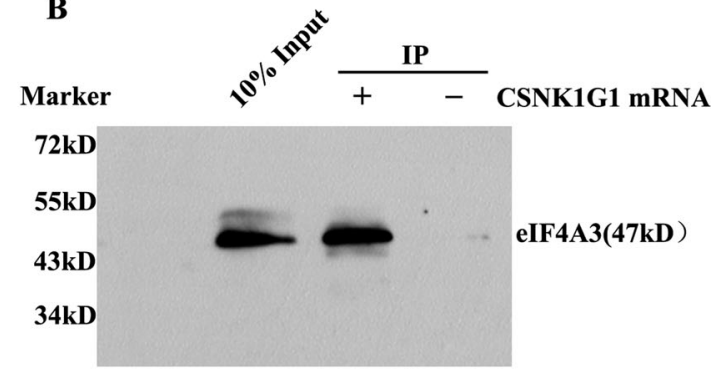

$\mathbf{C}$

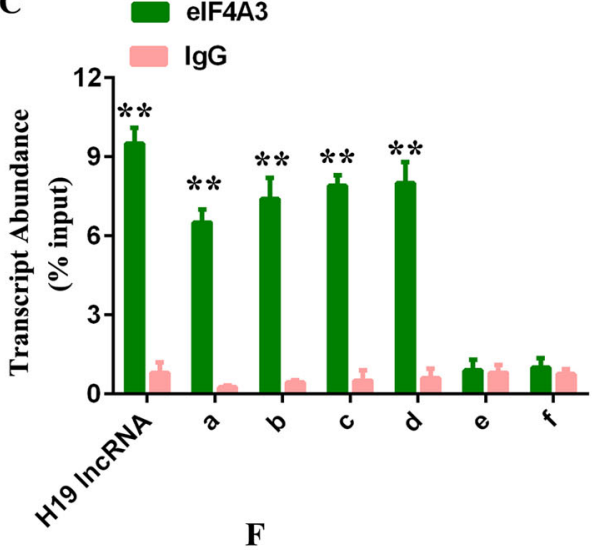

$\mathbf{E}$

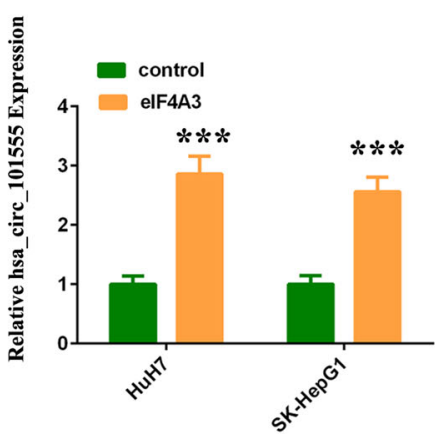

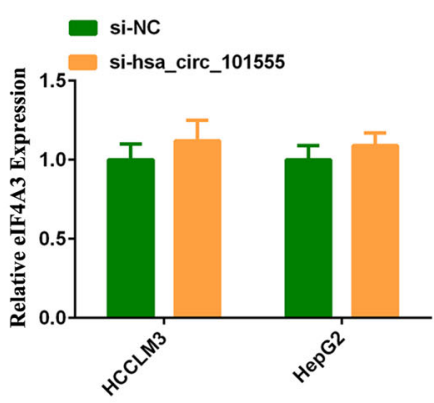

Fig. 4 The RNA-binding protein EIF4A3 regulates hsa_circ_101555 expression. A Binding sites for EIF4A3 on the CSNK1G1 mRNA transcript, upstream or downstream of hsa_circ_101555, obtained from Circular RNA Interactome. B Pull-down assay confirms the enrichment of ElF4A3 protein in CSNK1G1 mRNA pull-down. C Quantitative real-time PCR following EIF4A3-RIP assay confirms the binding of EIF4A3 on the predicted binding region ( $a, b$, c, and d) of CSNK1G1 mRNA. H19, a known interacting long noncoding RNA (IncRNA) with EIF4A3, served as the positive control. The intron 10 of CSNK1G1 mRNA was used as a negative control. D HCCLM3 and HepG2 cells transfected with control or siRNA targeted elF4A3; hsa_circ_101555 expression detected by qRT-PCR. E HCCLM3 and HepG2 cells transfected with control or elF4A3 overexpression plasmids; hsa_circ_101555 expression measured by qRT-PCR. F HCCLM3 and HepG2 cells transfected with control or siRNA targeted hsa_circ_101555; elF4A3 expression detected by qRT-PCR. 

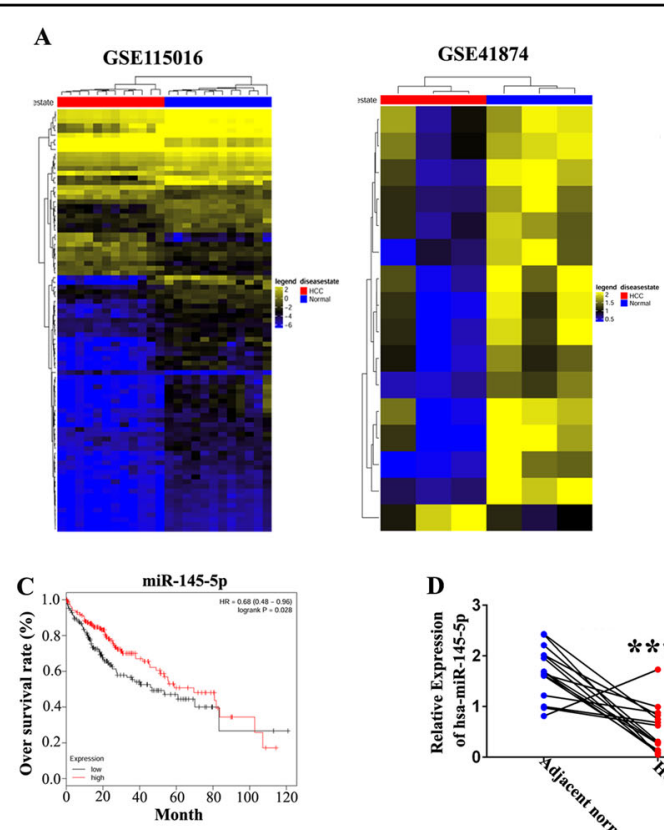

\section{F} miR-145-5p

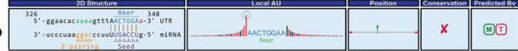

$$
\text { hsa-mik-145-5p }
$$

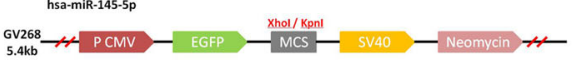

hsa-circ-101555

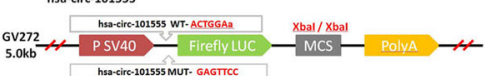

G
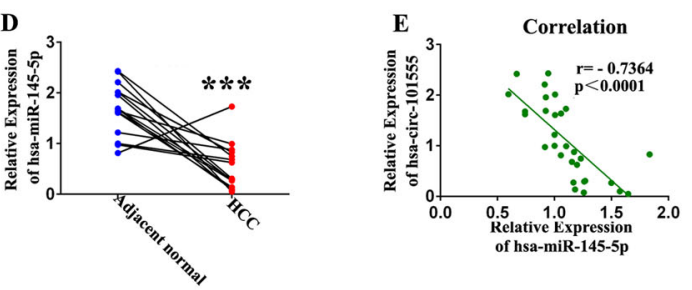
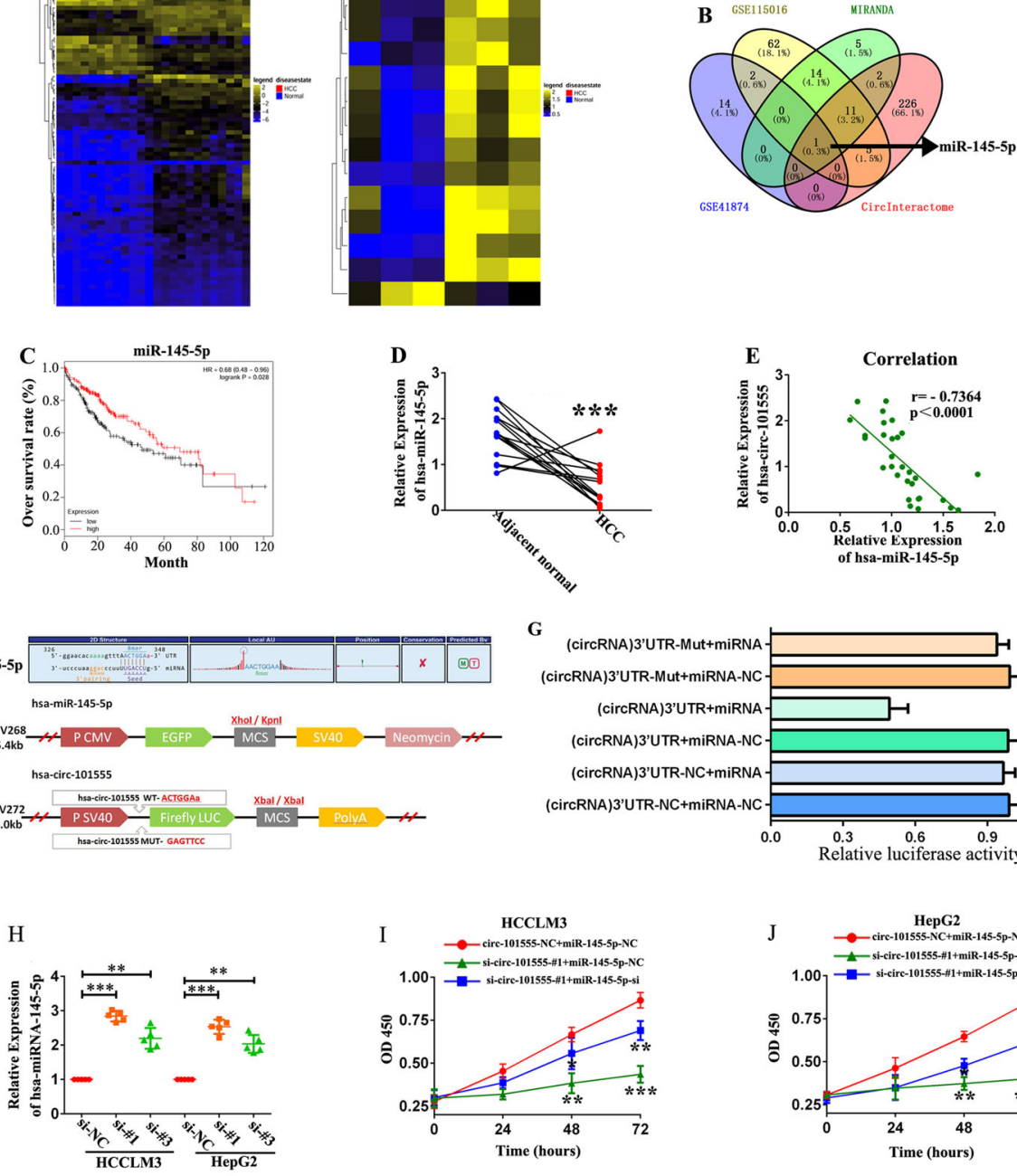

G (circRNA) 3'UTR-Mut+miRNA (circRNA)3'UTR-Mut+miRNA-N (circRNA) 3'UTR+miRNA (circRNA) 3'UTR+miRNA-NC (CirCRNA) 3'UTR-NC+miRNA (circRNA) 3'UTR-NC+miRNA-NC

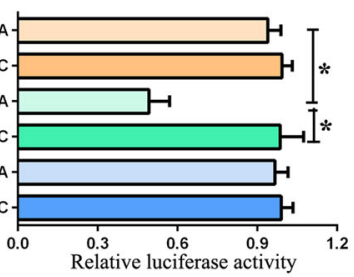

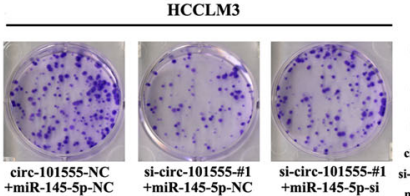

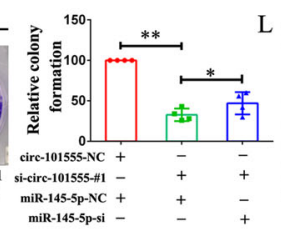

L

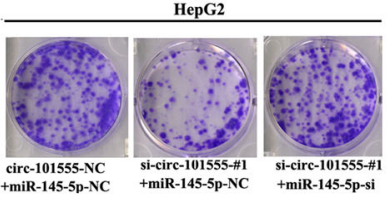

$\mathrm{N}$

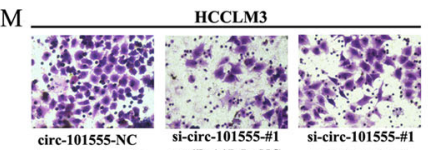

irc-101555-NC si-circ-101555-11 $+\mathrm{miR}-145-5 \mathrm{p}-\mathrm{NC}$
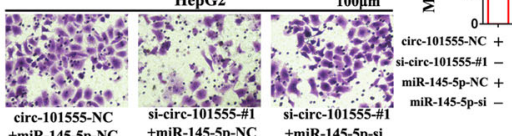

circ-101555-NC

+ miR-145-5p-NC

si-circ-10155-
+ miR-145-5p-si
(100-1 $\overline{100 \mu \mathrm{m}}$

HCCLM3

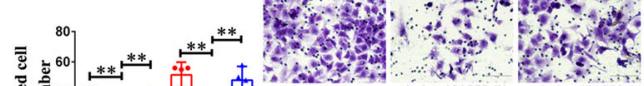

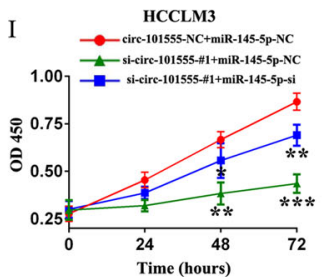
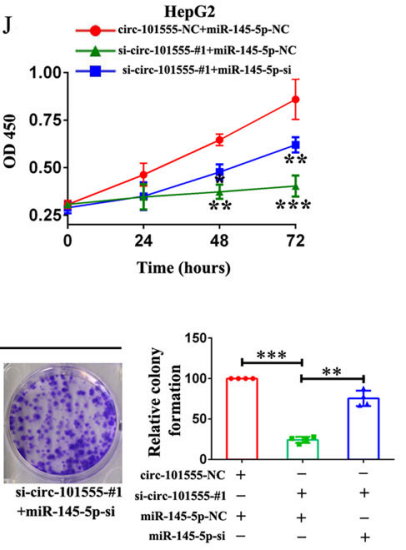

${ }_{\overline{8}}^{80}{ }^{80} 60$

***,

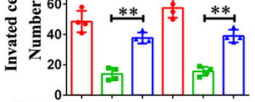

Fig. 5 (See legend on next page.) 
(see figure on previous page)

Fig. 5 Identification of miRNAs binding to hsa_circ_101555 that modulate the proliferation, cell cycle, migration, and invasion of HCC cells via miR-145-5p. A Heatmap analyses exhibit differentially expressed miRNAs in HCC with |log2fold-change $\mid>1.0$ and $P<0.05$. B Venn analysis between predicted miRNA targeted by hsa_circ_101555 and differentially expressed miRNAs implying that miR-145-5p is involved in HCC. C Kaplan-Meier curves imply that patients with low miR-145-5p levels have a poorer 10-year overall survival. D Expression of miR-145-5p in 10 HCC tumor tissues and adjacent normal tissues, measured by qRT-PCR ( $\left.{ }^{* *} P<0.001\right)$. E Pearson correlation analyses showing a correlation between hsa_circ_101555 and miR-145-5p expression $(n=20)$. F, G Schematic of hsa_circ_101555 wild-type (wt) and mutant (mut) luciferase reporter vectors. Luciferase reporter assay in HEK293T cells co-transfected with miRNA mimics, hsa_circ_101555-wt and mutant (mut) luciferase reporter vectors. H Expression of miR-145-5p analyzed by qRT-PCR in HCCLM3 and HepG2 cells transfected with siRNA-hsa_circ_101555-\#1, siRNA-hsa_circ_101555-\#3, or siRNA-NC (**P $\left.<0.01 ;{ }^{* * *} P<0.001 ; n=5\right)$. I, J Viability of HCCLM3 and HepG2 cells after co-transfection with siRNA-hsa_circ_101555 and miR-145$5 p$ inhibitor measured using WST-1 assays $\left({ }^{*} P<0.05 ;{ }^{* *} P<0.01 ;{ }^{* * *} P<0.001 ; n=4\right)$. K, L Cell proliferation ability of HCCLM3 and HepG2 cells COtransfected with siRNA-hsa_circ_101555 and miR-145-5p inhibitor evaluated by colony formation assay $\left(* P<0.05 ; * * P<0.01 ;{ }^{* * *} P<0.001 ; n=4\right)$. M, N Cell migration or invasion assays performed in HCCLM3 and HepG2 cells co-transfected with siRNA-hsa_circ_101555 and miR-145-5p inhibitor using transwell chamber, with or without Matrigel, respectively (**P<0.01; *** $<0.001 ; n=4)$.

(Fig. 5F-G). These findings indicate that hsa_circ_101555 may act as a sponge for miR-145-5p.

\section{hsa_circ_101555 modulates HCC cell proliferation, migration, and invasion via miR-145-5p}

We further investigated whether hsa_circ_101555 affects the function of $\mathrm{HCC}$ cells via miR-145-5p by determining its expression levels in vitro. qRT-PCR revealed that hsa_circ_101555 silencing increased miR-145-5p levels in HepG2 and HCCLM3 cells (Fig. 5H). We also found that the proliferation (via WST-1 assay) and colony-forming ability of HCC cells co-transfected with si-hsa_circ_101555_003 and miR-145-5p inhibitor were higher than those of HCC cells transfected with only siRNA-hsa_circ_101555, suggesting that the downregulation of miR-145-5p could partially reverse the proliferation-inhibitory effect of siRNAhsa_circ_101555 (Fig. 5I-L). Moreover, the decrease in HepG2 and HCCLM3 cell migration and invasion caused by hsa_circ_101555 silencing could be reversed by miR-145-5p inhibition (Fig. 5M-N). Collectively, these results demonstrate that hsa_circ_101555 was required to sustain HCC cell progression and partly functions by impairing the tumor suppressor miR-145-5p.

\section{Analysis of CDCA3 expression in HCC}

To better understand the regulatory mechanism of miR145-5p in HCC, we identified its potential target genes using mRNA array from GSE115019 and GSE84402 datasets (Fig. 6A). Potential miR-145-5p target mRNAs were predicted by bioinformatics (TargetScan and miRanda). We then identified four miR-145-5p-related genes (NTN4, CDCA3, SLC25A25, and SLC1A2) by Venn analysis between HCC-related and miR-145-5p predicted genes (Fig. $6 \mathrm{~B}$ and $\mathrm{C}$ ). Analysis of the overall survival of these miR-145-5p-related genes using GEPIA (http:// gepia.cancer-pku.cn/) revealed that high $C D C A 3$ levels were associated with poorer overall survival (Fig. 6D-G).

We further detected $C D C A 3 \mathrm{mRNA}$ expression in ten paired HCC and adjacent normal tissues by qRT-PCR to better understand its role and found it to be significantly upregulated in HCC tumors (Fig. 6H). Additionally, western blot and immunohistochemistry (IHC) analyses revealed that the protein expression of CDCA3 in HCC tissues was significantly upregulated as compared to their adjacent normal tissues (Fig. $6 \mathrm{I}$ and $\mathrm{J}$ ).

These results demonstrate that CDCA3 may represent a primary target of miR-145-5p. Pearson correlation analysis further revealed a negative correlation $(p=0.0004$, $r=-0.6065)$ between miR-145-5p and CDCA3 expression levels (Fig. 6K) and a positive correlation $(p<0.0001$, $\mathrm{r}=0.6544)$ between hsa_circ_101555 and CDCA3 expression levels (Fig. 6L).

\section{miR-145-5p directly targets the CDCA3 3'-UTR}

The miR-145-5p-CDCA3 interaction was confirmed via luciferase reporter assays, where in miR-145-5p significantly reduced the activity of the luciferase reporter compared to the negative control (wild-type CDCA3 sequence). However, such reduction was not observed following mutation of the miR-145-5p binding sites (Fig. 7A). These results indicate that miR-145-5p negatively regulated the expression of CDCA3.

Moreover, miR-145-5p inhibition significantly increased CDCA3 protein levels, and miR-145-5p mimetic agents reduced CDCA3 expression in HepG2 and HCCLM3 cells (Fig. 7B). Meanwhile, knockdown of hsa_circ_101555 significantly inhibited CDCA3 expression, while overexpression of hsa_circ_101555 had the opposite effect (Supplementary Fig. 7A and B). Additionally, downregulation of CDCA3 in HepG2 and HCCLM3 cells, caused by hsa_circ_101555 silencing, could be reversed by miR145-5p inhibition (Supplementary Fig. 7C). miR-145-5p inhibition also promoted the proliferation, migration, and invasion of HCC cells. Importantly, these enhancements were not observed in cells co-transfected with siRNACDCA3 and miR-145-5p inhibitor (Fig. 7C-H), suggesting that miR-145-5p targets CDCA3 and subsequently inhibits the proliferation, migration, and invasion of HCC cells. 


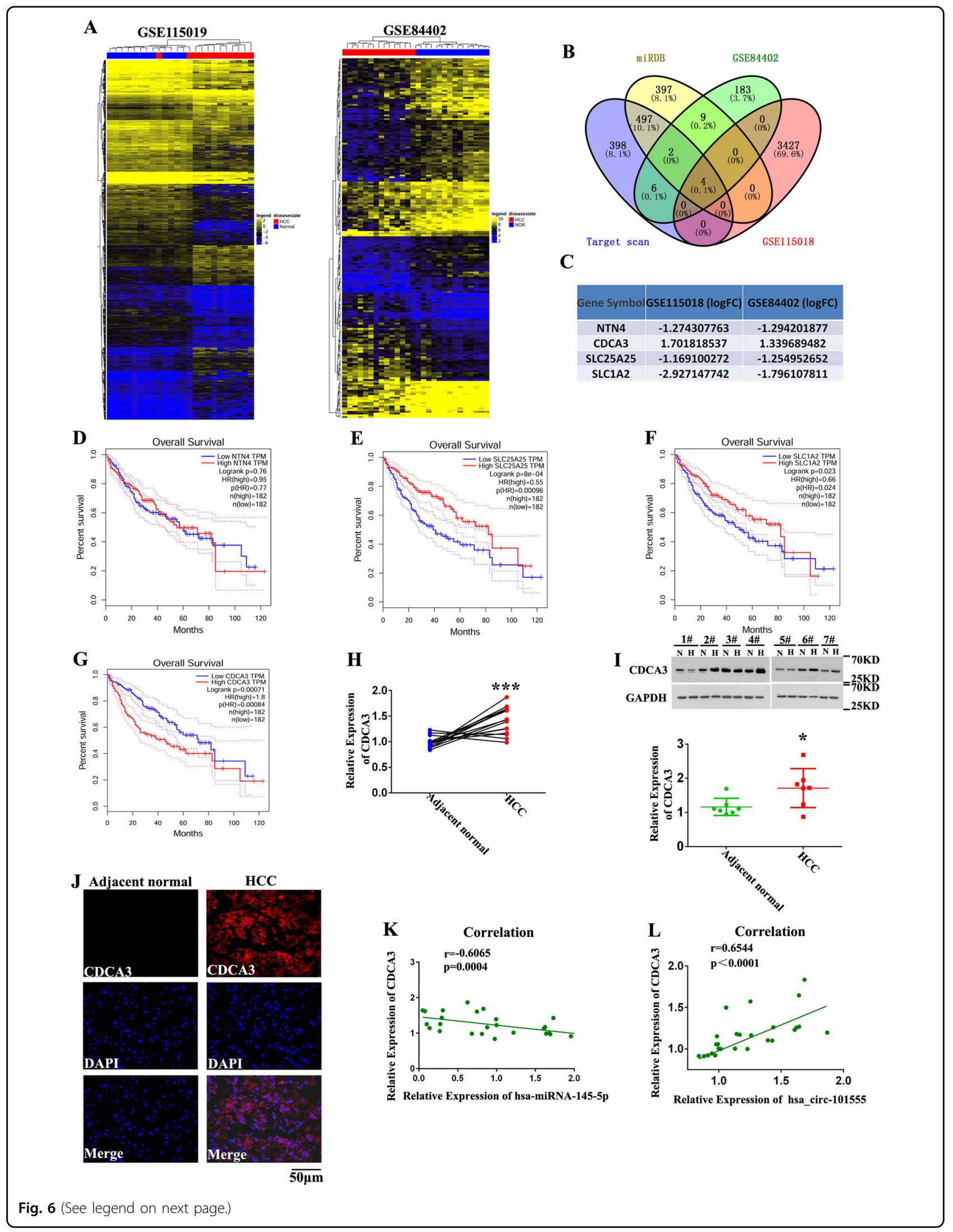


(see figure on previous page)

Fig. 6 Identification of CDCA3 as an miRNA target oncogenes. A Heatmap analyses demonstrating differentially expressed mRNAs in HCC with | log2fold-change $\mid>1.0$ and $P<0.05$. B, C Venn analysis between predicted mRNA genes targeted by miR-145-5p and differentially expressed mRNAs identified four mRNAs, NTN4, CDCA3, SLC25A25, and SLC1A2 involved in HCC. D-G CDCA3 is significantly upregulated in HCC tumors and is associated with poorer OS. $\mathbf{H}$ Expression of CDCA3 between ten HCC tumor tissues and adjacent normal tissues measured by qRT-PCR $(* * * P<0.001, n=10)$. I Expression of CDCA3 between seven HCC tumor and adjacent normal tissues measured by western blot $(* * P<0.01 ; n=7)$. J CDCA3 protein expression in HCC and corresponding adjacent normal tissues detected by $\mathrm{HCC}(n=3)$. $\mathbf{K}$ Pearson correlation analysis demonstrating correlation between miR-145-5p and CDCA3 expression $(n=20)$. L Pearson correlation analysis showing the correlation between hsa-circ-101555 and CDCA3 expression $(n=20)$.

hsa_circ_101555 is required to sustain HCC tumor growth in vivo

Considering that study demonstrated that hsa_circ 101555 knockdown suppressed the viability of HepG2 and HCCLM3 cells, we further examined its role in HCC tumorigenesis in vivo. Figure $8 \mathrm{~A}$ provides a schematic diagram of tumor xenograft generation and subsequent treatment with cholesterol-modified hsa_circ_101555 shRNA or negative control shRNA. Body weight and tumor volume measurements, taken every 3 days for 33 days, revealed that mice receiving hsa_circ_101555 shRNA exhibited a marked decrease in tumor volume and weight compared to those receiving control shRNA (Fig. $8 \mathrm{~B}-\mathrm{F}$ ). This suggested that hsa_circ_101555 promoted HCC cell growth in vivo. These results further supported a role for hsa_circ_101555 in HCC tumorigenesis and development.

Moreover, immunohistochemical staining of xenografted tumors demonstrated that hsa_circ_101555 silencing significantly reduced the Ki-67 proliferation index (Fig. 8G). Hsa_circ_101555 silencing also significantly inhibited hsa_circ_101555 and CDCA3 expression, while increasing miR-145-5p expression in the hsa_circ_101555-silenced group compared to the negative control group (Fig. 8H-J). Western blotting further demonstrated that hsa_circ 101555 silencing significantly reduced CDCA3 abundance (Fig. 8K), which was supported by immunohistochemical staining of xenografted tumors (Fig. 8L).

\section{Discussion}

In the present study we identified 36 circRNAs with differential and consistent expression in GSE94508, GSE97322, and GSE78520 datasets. As hsa_circ_101555 was the only upregulated circRNA appearing in the three GSE datasets, it became the focus of further analyses. We characterized hsa_circ_101555 in HCC cells, and found that it consisted of six exons (exon 1-6, $815 \mathrm{nt}$ ) and was derived from the host gene CSNK1G1. Moreover, it was cyclized with the head-to-tail splicing of exon 1 and exon 6 and resisted RNase $R$ treatment, demonstrating its stability compared to linear RNA.

Previous studies have shown that circRNAs play an essential role in cell cycle progression and proliferation ${ }^{26-29}$. hsa_circ_101555 was specifically reported as upregulated in tumor tissues and as associated with the prognosis of colorectal cancer patients, while its silencing significantly suppresses cell proliferation, induces apoptosis, and impairs the DNA repair capacity of CRC cells ${ }^{30}$. Herein, we found that hsa_circ_101555 was highly expressed in HCC cell lines (most markedly in HCCLM3) as well as patient tissues compared to adjacent nontumor tissues. More importantly, in a murine xenograft model, hsa_circ_101555 silencing significantly reduced HCC growth. We also provided evidence that the ectopic expression of hsa_circ_101555 is likely required to sustain cell proliferation. Meanwhile, we previously demonstrated that circMAST1 silencing inhibits HCC cell migration and invasion, which are important determinants of tumor metastasis ${ }^{31}$. Our results are consistent with those of previous studies that showed a regulatory role for circRNAs in cancer proliferation, migration, and invasion ${ }^{32-36}$. Thus, our research confirmed the stable role of hsa_circ_101555 in promoting HCC progression. Although the mechanisms through which circRNAs regulate carcinogenesis and cancer progression have not been fully elucidated, the "circRNA-miRNA-mRNA" axis, also known as the "miRNA sponge," is frequently cited ${ }^{37}$. In the present study, we confirmed that hsa_circ_101555 is an miR-145-5p sponge, evidenced by the significant increase in miR-145-5p expression following silencing of hsa_circ_101555, which in turn inhibited the proliferation, migration, and invasion of HCC cell lines. We also confirmed a direct correlation between miR-145-5p and hsa_circ_101555 expression. Consistent with our findings, several other studies have shown that circRNAs act as miRNA sponges during the development and progression of HCC. Hu et al. reported that circASAP1 acts as a ceRNA for miR-326 and miR-532$5 \mathrm{p}$, which are tumor suppressors that regulating cancer cell proliferation, colony formation, migration, and invasion ${ }^{38}$. Further, many miRNAs have been shown to play critical roles in HCC initiation, development, and progression ${ }^{39-43}$. miR-145-5p is a tumor suppressor that is downregulated in several cancer types including glioma ${ }^{44}$, upper tract urothelial carcinoma ${ }^{45}$, and gastric cancer ${ }^{46}$. Consistent with these reports, our findings indicate that miR-145-5p serves as a tumor suppressor miRNA in HCC. As hsa_circ_101555 sponges miR-145-5p, the increased expression of hsa_circ_101555 in HCC cells leads to a 


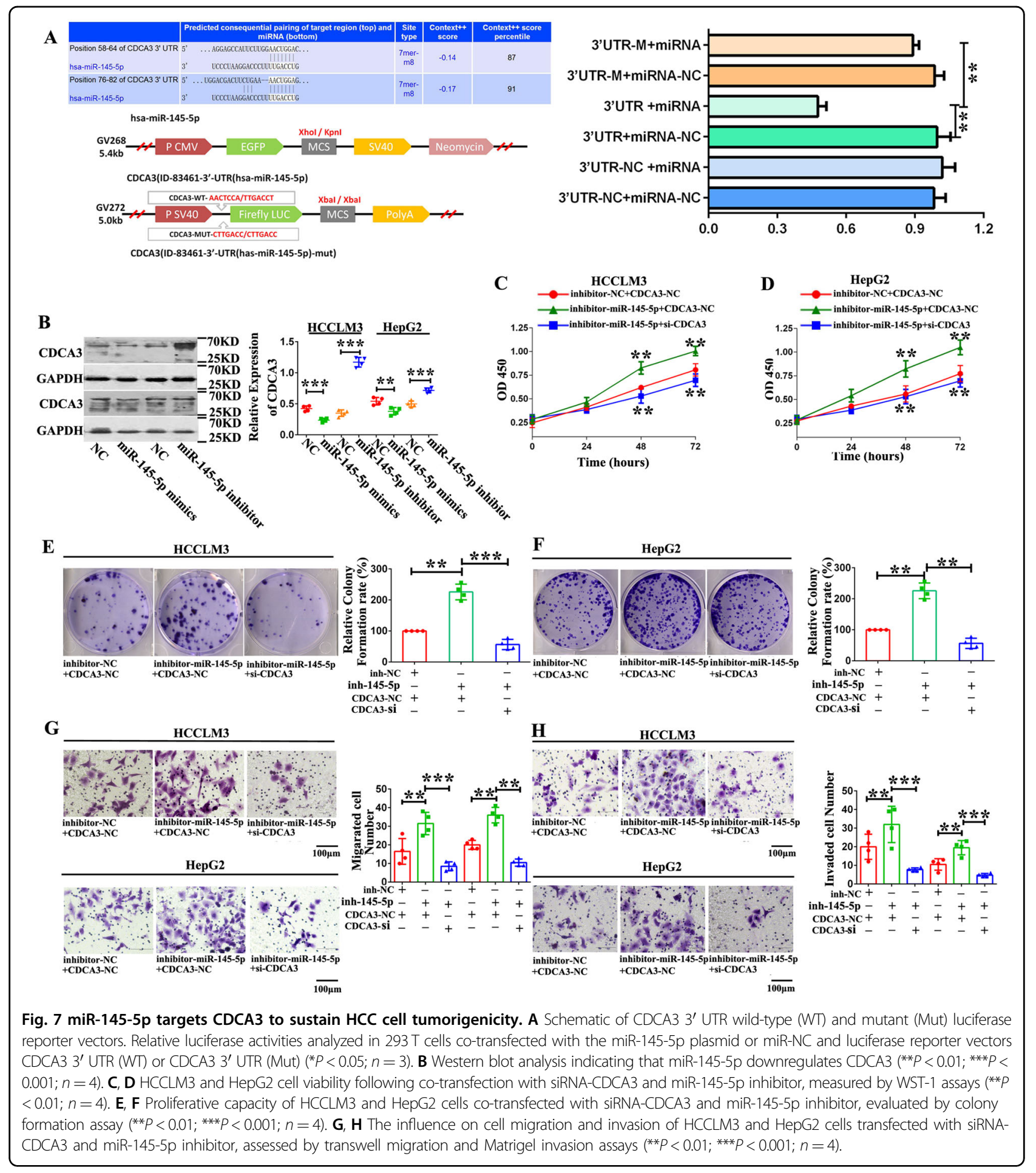

decrease in miR-145-5p expression, thereby promoting cancer cell proliferation, migration, and invasion. Conversely, inhibiting hsa_circ_101555 expression increased miR-145-5p, which consequently suppressed the proliferation, migration, and invasion of HCC cells. However, we found that simultaneous inhibition of both hsa_circ_101555 and miR-145-5p expression, resulted in increased tumorigenic properties in HCC cells compared to that in cells with only hsa_circ_101555 inhibition. Thus, our results provide evidence that hsa_circ_101555 regulates HCC progression via miR-145-5p sponging, and that hsa_circ_101555 is an upstream target of miR-145-5p. 
A

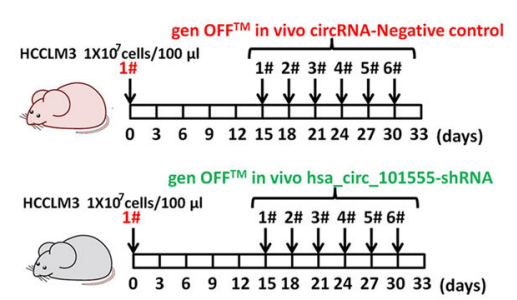

D

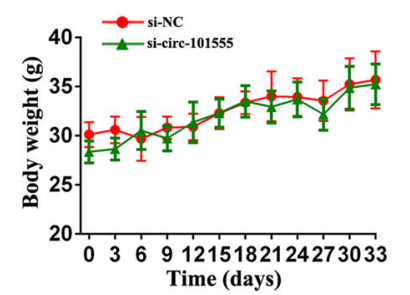

G

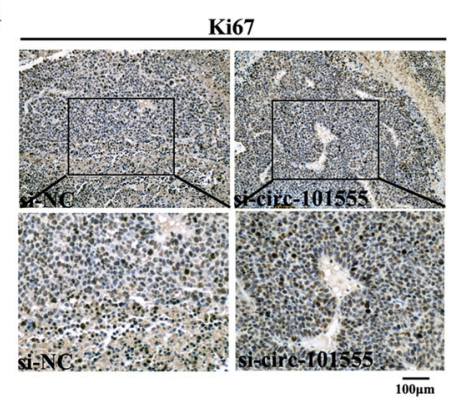

I

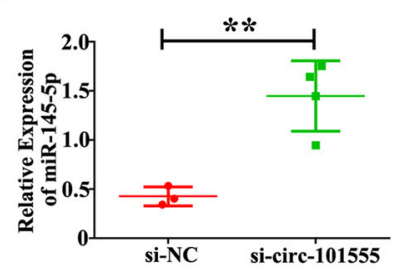

$\mathbf{L}$
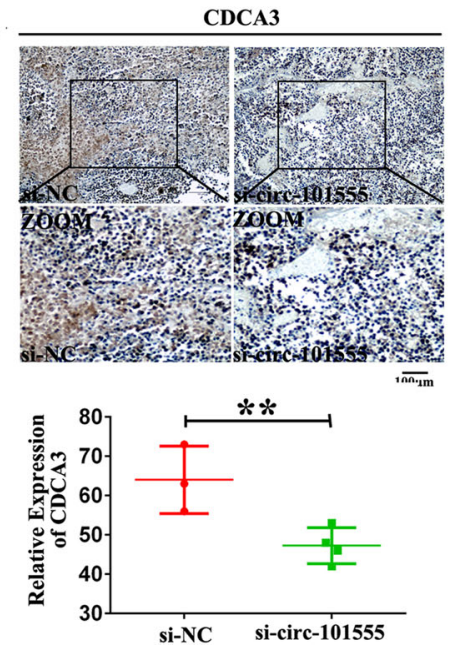

B

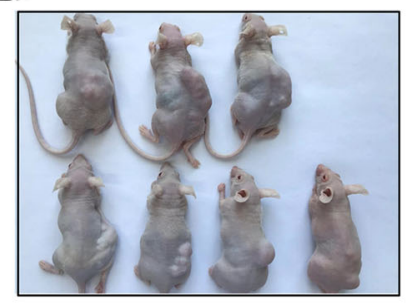

$\mathbf{E}$
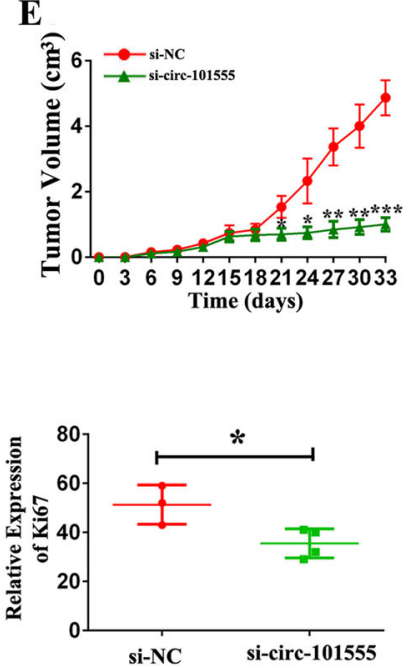

$\mathbf{J}$

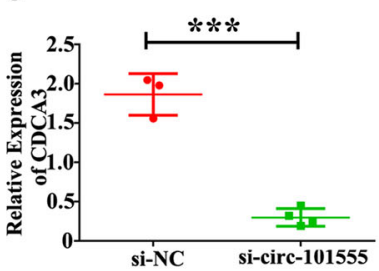

C

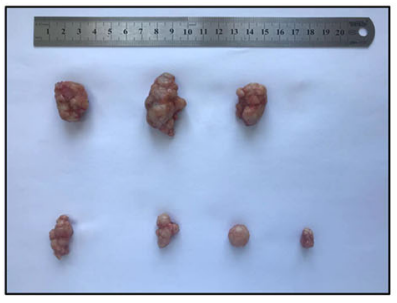

F

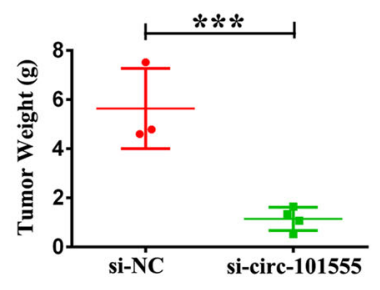

H

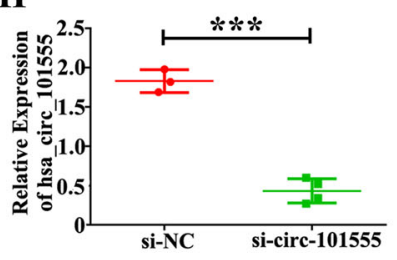

$\mathrm{K}_{\text {CDCA3 }} \frac{\text { si-NC }}{\text { si-circ-101555 }}$

GAPDH $---0--{ }_{-25 \mathrm{KD}}^{25 \mathrm{KD}}$
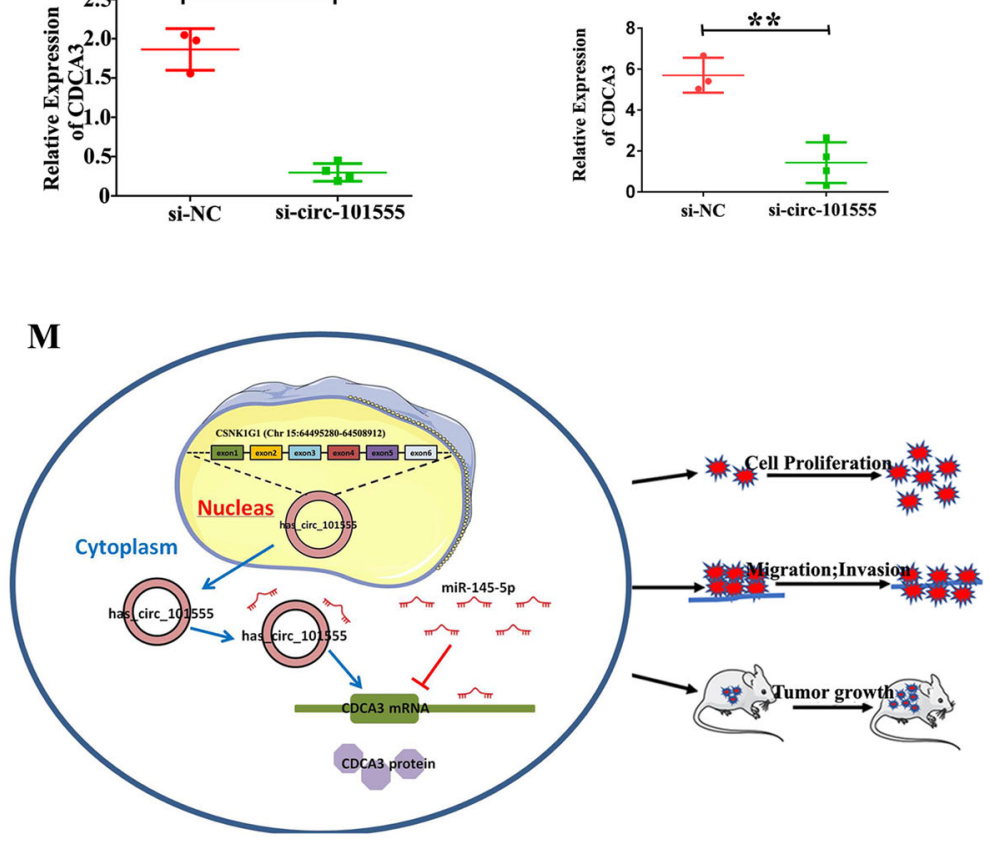

Fig. 8 (See legend on next page.) 
(see figure on previous page)

Fig. $\mathbf{8}$ hsa_circ_101555 promotes HCC tumor growth in vivo. A Schematic diagram shows that HCCLM3 cells were injected subcutaneously into BALB/C nude mice for 15 days to create tumor xenografts. After 15 days, 10 nmol cholesterol-modified hsa_circ_101555 shRNA or negative control shRNA was subcutaneously injected at the tumor site every 3 days for 18 days. B Representative images of HCC tumor bearing BALB/C nude mice. C Body weight of xenograft nude mice. D Body weight measured every 3 days. E Tumor volumes measured every 3 days $\left({ }^{*} P<0.05 ;{ }^{* *} P<0.01\right.$; $\left.{ }^{* * *} P<0.001 ; n=4\right)$. F Relative weights of tumors ( $\left.{ }^{* *} P<0.001 ; n=4\right)$. G $I H C$ staining and $I H C$ analysis of Ki-67 expression in subcutaneous xenograft tumors. Knockdown of hsa_circ_101555 downregulates Ki-67 expression $(* P<0.05 ; n=4)$. H-J Expression of hsa_circ_101555, miR-145-5p and CDCA3 in subcutaneous xenograft tumors $\left({ }^{* *} P<0.01 ;{ }^{* * *} P<0.001 ; n=4\right)$. $\mathbf{K}$ Protein expression level of CDCA3 in seven paired HCC and normal tissues ${ }^{* *} P<$ $0.01 ; n=4)$. $\mathbf{L} I H C$ staining and $I H C$ analysis of CDCA3 expression in subcutaneous xenograft tumors. Knockdown of hsa_circ_101555 downregulates CDCA3 expression ( $\left.{ }^{* *} P<0.01 ; n=4\right)$. $\mathbf{M}$ Schematic diagram shows that hsa_circ_101555 promotes HCC cells proliferation, migration, and invasion through the miR-145-5p/CDCA3 axis.

The role of miRNA sponging in tumor progression has previously been described ${ }^{47}$. We confirmed, via bioinformatics and luciferase reporter gene analyses, that hsa_circ_101555-miR-145-5p targets the oncogene $C D C A 3$. Although several studies have implicated $C D C A 3$ in the regulation of cancer development and progres$\operatorname{sion}^{48-50}$, to our knowledge, this is the first study to confirm $C D C A 3$ expression in liver cancer tissues and report its positive correlation with hsa_circ_101555 expression and negative correlation with miR-145-5p expression. Meanwhile, a previous study in colorectal cancer demonstrated that miR-145-5p suppressed proliferation, metastasis, and epithelial-mesenchymal transition by targeting $C D C A 3^{51}$. Our results were consistent with these reports. In addition, we found that the miR$145-5$ p/CDCA3 axis is regulated by hsa_circ_101555 via a sponging mechanism. Further, we demonstrated that miR-145-5p suppression promotes CDCA3 expression, which in turn increases the proliferation, migration, and invasion of HCC cells. The simultaneous inhibition of miR-145-5p and CDCA3 attenuates the tumorigenic features of HCC cells to a greater extent than miR-145-5p inhibition alone. To our knowledge, our study is the first to demonstrate hsa_circ_101555 involvement in regulation of $C D C A 3$ expression. Moreover, we demonstrated that hsa_circ_101555 silencing significantly reduces $C D C A 3$ expression in vivo. These findings suggest that hsa_circ_101555 protects CDCA3 from miR-145-5pmediated degradation in a ceRNA-mediated manner.

The biogenesis of circRNAs is regulated by specific cisacting elements and trans-acting factors. It has been shown that certain RNA-binding proteins promote circRNA expression ${ }^{52}$. RNA-binding protein EIF4A3 is the core component of exon junction complex (EJC), which is considered as an important regulator of posttranscriptional regulation processes including mRNA splicing, transport, translation, and surveillance ${ }^{53}$. Through bioinformatic analysis and experiments, we predicted and screened that EIF4A3 could bind to a flanking sequence of hsa_circ_101555. Our research reveals that EIF4A3-mediated reverse splicing of exons can be a potential mechanism to induce high expression of hsa_circ_101555.

We acknowledge that our research has certain limitations. Although this study clarifies that hsa_circ_101555 functions as a sponge of miR-145-5p to promote CDCA3induced HCC cancer cell proliferation and invasion, circRNAs may regulate the development and progression of HCC via other mechanisms. For example, circRNAs have been shown to regulate parental gene expression and the expression of peptides/proteins in other cancers $^{36,54}$. Thus, additional research is required to further explore the role of hsa_circ_101555 in HCC. In terms of clinical diagnosis and treatment, we additional studies are required, including expanding the sample size and expression stability of hsa_circ_101555 in the peripheral blood of HCC patients, as well as evaluating the initiation of its high expression among the stages of HCC.

To summarize, we found that: (1) hsa_circ_101555 is highly expressed in HCC tissues and HCC cell lines (HepG2 and HCCLM3); (2) silencing hsa_circ_101555 in a murine xenograft model significantly reduces the growth of HCC; (3) hsa_circ_101555 is likely required to sustain proliferation, migration, and invasion in HCC cell lines; (4) hsa_circ_101555 acts as an miR-145-5p sponge, while silencing hsa_circ_101555 significantly inhibits cell growth; (5) hsa_circ_101555 sponges miR-145-5p to promote CDCA3 expression; and (6) eIF4A3 induces hsa_circ_101555 cyclization and increases hsa_circ_101555 expression. Thus, our study identified a previously unrecognized role for hsa_circ_101555 in sustaining HCC progression.

\section{Conclusions}

Our study demonstrated that hsa_circ_101555 is upregulated in HCC cell lines and patient tissues, and its high expression is associated with HCC progression. Moreover, hsa_circ_101555 functions as a tumor promotor and is required to sustain the proliferation and invasion of $\mathrm{HCC}$ by directly binding to miR-145-5p and impeding its suppression of CDCA3. Furthermore, we also demonstrate that EIF4A3 could mediate the biogenesis of hsa_circ_101555, 
but the detailed mechanism needs further study. Based on its role in regulating the miR-145-5p/CDCA3 axis, our findings suggest that hsa_circ_101555 may represent a potential novel biomarker and therapeutic target for HCC.

\section{Materials and methods}

\section{Gene Expression Omnibus dataset}

We downloaded the expression microarray data (CEL data) from the GSE7852, GSE94508, GSE97322, GSE115016, GSE41874, GSE115018, and GSE84402 dataset of the GEO (http://ncbi.nlm.nih.gov/geo/). Detailed expression profiles are provided in Additional file 1-7.

\section{Cell lines and clinical tissues}

A total of 38 HCC samples were obtained from the clinical sample bank of the First Affiliated Hospital, Harbin medical university. The collection of human specimens was approved by the Biomedical Ethics Committee of the Harbin medical university First Affiliated Hospital and written informed consent was obtained from each patient (HMUDQ20200010202). Inclusion criteria for patient selection was curative hepatectomy performed between 2017 and 2018. All patients were pathologically diagnosed with hepatocellular carcinoma and liver specimens were evaluated by pathologists to determine clinical staging according to the TNM classification. HCC patients with the following conditions were excluded: (1) patients $\leq 18$ or $\geq 70$ years of age or without full civil capacity; (2) patients with a history of preoperative anticancer radiotherapy or chemotherapy, biological, immune, or traditional Chinese medicine administration; (3) patients with incomplete postoperative follow-up data; (4) patients with a history of another organ malignancy, or systemic immune disease. All specimens were collected within $15 \mathrm{~min}$ of removal from the body and were immediately snap-frozen in liquid nitrogen before storage at $-80^{\circ} \mathrm{C}$. Ten paired samples were used to compare the expression levels of the genes of interest between $\mathrm{HCC}$ and paired nontumorous tissues. The detailed clinicopathological features, as well as the correlations between hsa_circ_101555 expression and the clinical characteristics, are described in Additional files 8-9: Tables S1-S2.

Cell lines used in this study (HepG2, HCCLM3, and SKHep1) were purchased from the Cell Bank of Type Culture Collection (Chinese Academy of Sciences, Shanghai, China). Huh-7 and L02 were purchased from the Procell (Wuhan, China). All cells were cultured in DMEM/high glucose medium (Hyclone, Logan, UT, USA) supplemented with $10 \%$ fetal bovine serum (PAN-Biotek, Aidenbach, Bavaria) and $1 \%$ penicillin-streptomycin (Hyclone) in a humidified atmosphere at $37^{\circ} \mathrm{C}$ containing $5 \% \mathrm{CO}_{2}$.

\section{Xenograft nude mouse model}

Six-week-old male BALB/C nude mice purchased from Vital river (Beijing, China) were maintained under specific pathogen-free conditions with a 12-h light/dark cycle. All animal experiments were performed in accordance with a statement of compliance with ethical regulations and approved by the Biomedical Ethics Committee of the Harbin medical university. Animals are grouped randomly during the experiment. HCCLM3 cells were subcutaneously injected into the right upper back of the nude mice $\left(1 \times 10^{6}\right.$ cells per mouse) for 15 days, after subcutaneous incubation of HCC tumor mass. Next, $10 \mathrm{nmol}$ cholesterol-modified hsa_circ_101555 shRNA or control shRNA RiboBio (Guangzhou, China) were intratumorally injected every 3 days for 18 days. the last day of the injections, which would account for day 33 of the experiment, the mice were sacrificed and tumor tissues were collected for examination.

\section{Circular structure confirmation}

The circular structure of hsa_circ_101555 was confirmed by Sanger sequencing, divergent primer PCR and RNase $\mathrm{R}$ treatment. PCR products, amplified by divergent primers of hsa_circ_101555, were inserted into the $\mathrm{T}$ vector and delivered to SinoGENE (Beijing, China) for Sanger sequencing. The results were crosschecked with the back-spliced region of hsa_circ_101555 supplied by circBASE $^{55}$. Total RNA was extracted from HCC tissue using Trizol solution. For RNase R treatment, $3 \mu \mathrm{g}$ of total RNA extracted from HCC was incubated with $10 \mathrm{U}$ RNase R (20 U/ $\mu \mathrm{L}$, Epicentre, Madison, WI, USA) in a $10 \mu \mathrm{L}$ total volume at $37^{\circ} \mathrm{C}$ for $45 \mathrm{~min}$, followed by incubation at $70^{\circ} \mathrm{C}$ for $10 \mathrm{~min}$ to deactivate RNase R. The treated RNAs were used for qRT-PCR ${ }^{27}$.

\section{Quantitative real-time polymerase chain reaction, western blotting, and immunofluorescence analyses}

Total RNA was extracted from HCC cell lines and tissue using Trizol solution, and complement DNA was generated using the Golden 1st cDNA Synthesis kit (Haigene, China) following RNA quantification. qRT-PCR assays were performed using Power SYBB Green PCR Master Mix (Life Technologies, Carlsbad, CA, USA). The circRNA and gene expression levels were normalized to that of $G A D P H$, while miRNA expression levels were normalized to that of U6. Each sample was tested in triplicate. The relative expression was analyzed by the comparative cycle threshold $(\mathrm{Ct})$ method, according to the equation $2^{-\Delta \Delta \mathrm{Ct}}\left[\Delta^{\mathrm{Ct}}=\mathrm{Ct}-\mathrm{Ct}(\mathrm{GAPDH})\right]$. The primer sequences of hsa_circ_101555 and U6 were designed by RiboBio (Guangzhou, China). The linear101555, CDCA3, miR-145-5p, and GAPDH sequences were designed by Genscript (Nanjing, China). All experiments were performed in triplicate. The primers used in this study are 
listed in Additional file 10: Table S3. RNase R digestion was performed using RNase R purchased from Epicentre Biotechnologies (Madison, WI, USA) as per the manufacturer's instructions. Briefly, $1 \mu \mathrm{g}$ of extracted RNA was treated with $3 \mathrm{U}$ of RNase R (Epicenter, USA) and incubated at $37^{\circ} \mathrm{C}$ for $1 \mathrm{~h}$, after which the RNA was purified by phenol-chloroform extraction and subjected to qRT-PCR.

For western blotting, the total protein extracts from cells were separated by sodium dodecyl sulfate-polyacrylamide gel electrophoresis (SDS-PAGE), transferred onto polyvinylidene difluoride membranes, and incubated with the corresponding antibodies. The membranes were developed using the enhanced chemiluminescence method (Haigene, China). All antibodies used in this study are listed in Additional file 11: Table S4.

Fresh samples were cut to an appropriate size and fixed in $4 \%$ paraformaldehyde for $24 \mathrm{~h}$. The fixed specimens were dehydrated in a graded series of ethanol solutions, embedded in paraffin and cut at a thickness of $4 \mu \mathrm{m}$. The sections were dewaxed and rehydrated using xylene and ethanol, and high-pressure heat was applied for antigen retrieval. The sections were incubated with the primary antibodies overnight at $4{ }^{\circ} \mathrm{C}$. Finally, all sections were dehydrated, cleared, mounted, and visualized with a diaminobenzidine-based colorimetric method. The antibodies used in this study are listed in Additional file 11: Table S4.

\section{Fluorescence in situ hybridization}

In situ hybridization was performed with a FISH kit (RiboBio, Guangzhou, China). Cells, frozen HCC sections, and paired adjacent liver tissues were briefly rinsed in PBS and fixed in $4 \%$ formaldehyde for $10 \mathrm{~min}$. The cells were then permeabilized in PBS containing 0.5\% Triton X 100 at $4{ }^{\circ} \mathrm{C}$ for $5 \mathrm{~min}$, washed with PBS three times for $5 \mathrm{~min}$, and prehybridized at $37^{\circ} \mathrm{C}$ for $30 \mathrm{~min}$ before hybridization. Next, an anti- hsa_circ_101555, anti-U6, or anti-18S oligodeoxynucleotide probe (RiboBio, Guangzhou, China) was used in the hybridization solution at $37^{\circ} \mathrm{C}$ overnight in the dark. The next day, cells were counterstained with DAPI and imaged using a NA1.4 inverted Leica DMI6000 microscope (Leica, Heidelberg, Germany). Images were captured using a Hamamatsu ORCA-R2 camera (Hamamatsu, Japan) and recorded using LAS AF software (Leica). The experiments were conducted in triplicate.

\section{Cell proliferation, migration, and Matrigel invasion assay}

Cell proliferation was assessed using the WST-1 assay (Beyotime Biotechnology, Nantong, China). Cells $\left(2 \times 10^{3}\right)$ were seeded into each well of 96 -well plates and $10 \mu \mathrm{L}$ of WST-1 solution was added to each well at four timepoints $(0,24,48$, and $72 \mathrm{~h})$. After $4 \mathrm{~h}$ of incubation at $37^{\circ} \mathrm{C}$, the absorbance at $450 \mathrm{nM}$ was measured using a Spectra Max 250 spectrophotometer (Molecular Devices, Sunnyvale,
CA, USA). Experiments were independently performed in triplicate.

For the colony formation assays, cells $\left(1 \times 10^{2}\right)$ were suspended and plated into each well of 6-well plates. After 14 days incubation at $37^{\circ} \mathrm{C}$ in a chamber with an atmosphere of $5 \% \mathrm{CO}_{2}$, colonies were fixed with $1 \mathrm{~mL}$ of $4 \%$ paraformaldehyde (Solarbio, Beijing, China) for $30 \mathrm{~min}$ and stained with crystal violet (Beyotime Biotech-nology, Nantong, China) for $25 \mathrm{~min}$. Colonies were then counted after photographing the sample (Nikon, Tokyo, Japan).

Cell migration and invasion were measured using a transwell migration assay and a Matrigel invasion assay. For the transwell migration assay, $2-4 \times 10^{5}$ cells were suspended in $200 \mu \mathrm{L}$ of DMEM without serum and placed in the cell culture insert ( $8 \mu \mathrm{m}$ pore size; BD Falcon, San Jose, CA) of a companion plate (BD Falcon) with prewarmed culture medium containing $20 \%$ fetal bovine serum. The cells were incubated for $24 \mathrm{~h}$ at $37^{\circ} \mathrm{C}$ in $5 \%$ $\mathrm{CO}_{2}$ and subsequently fixed with $4 \%$ paraformaldehyde in PBS.

For the Matrigel invasion assay, $2-4 \times 10^{5}$ cells were suspended in $200 \mu \mathrm{L}$ of DMEM without serum and were placed in the cell culture insert precoated with $50 \mu \mathrm{L}$ Matrigel (BD Biosciences, San Jose, CA, USA). A prewarmed culture medium containing $20 \%$ fetal bovine serum was added to the well. The cells were incubated for $48 \mathrm{~h}$ at $37^{\circ} \mathrm{C}$ in $5 \% \mathrm{CO}_{2}$ and were then fixed with $4 \%$ paraformaldehyde in PBS. The nonmigrated or invaded cells on the top of the membrane were gently removed with a cotton swab. Cell migration or invasion was determined by staining cells with $0.1 \%$ crystal violet (Sigma, St Louis, MO) and the cells were counted under a light microscope ( $\times 200$ magnification) in eight randomly selected areas.

\section{Transfection experiment}

siRNA specific for hsa_circ_101555 was synthesized by RiboBio (Guangzhou, China), while the inhibitor of the miR-145-5p mimics and negative control, miRNA-1455p, siRNA of CDCA3 were synthesized by Gene Pharma (Shanghai, China). HepG2 and HCCLM3 cells were transfected with siRNA of hsa_circ_101555 or Huh-7 and SK-hep1 cells were transfected with lv-hsa_circ_101555 using the Lipofectamine 2000® siRNA transfection reagent (Invitrogen, Carlsbad, CA, USA) following the manufacturer's protocol. The target sequences of siRNAs are listed in Additional file 12: Table S5.

\section{Dual luciferase assay}

Targeted binding of hsa_circ_101555 to miR-145-5p was predicted using bioinformatics websites, including CircInteractome, and miRanda; whereas targeted binding of miR-145-5p to CDCA3 was predicted using TargetScan, and miRDB. The full-length sequences of hsa_circ_101555, 
with and without mutated predicted miR-145-5p binding sites, were subcloned into pmirGLO reporter vector (Promega, WI, USA). The full-length sequences of CDCA3, with and without mutated predicted miR-145-5p binding sites, were subcloned into pmirGLO reporter vector (Promega, WI, USA). Lipo2000 was then used for transfection of the vectors into $293 \mathrm{~T}$ cells. Finally, luciferase activity was measured using the dual luciferase assay kit.

\section{RNA pull-down}

RNA pull-down technology used desulphurized biotinlabeled RNA of CSNK1G1 mRNA and streptavidinlabeled magnetic beads to efficiently enrich and identify the RNA-binding protein, EIF4A3. RNA probes were labeled with biotin by in vitro transcription, and then incubated with cytoplasmic protein extract to form RNAprotein complexes. This complex was then allowed to bind streptavidin-labeled magnetic beads to separate them from the other components in the solution. After elution, western blotting was used to detect the binding proteins of EIF4A3 that interacted with CSNK1G1 mRNA.

\section{RIP assay}

RIP (RNA-binding protein immunoprecipitation) assay was performed with the BersinBio ${ }^{\mathrm{TM}}$ RNA Immunoprecipitation kit (Guangzhou, China). Cells were collected and cultivated in RIP lysis buffer, followed by immunoprecipitation with EIF4A3 antibody (Abcam, MA, USA). The final retrieved RNA was subjected to quantitative realtime PCR analysis. Normal mouse immunoglobulin G (IgG) served as negative controls.

\section{Statistical analyses}

All statistical analyses were performed using SPSS version 21.0 (IBM SPSS Inc., Chicago, IL, USA) and GraphPad Prism version 6.0 (GraphPad Software, La Jolla, CA, USA) software. Categorical variables are expressed as a count or percentage and tested using Chi-squared or Fisher's exact tests, as appropriate. Continuous data are reported as mean \pm standard deviation (SD) and compared using Student's $t$-test, one-way analysis of variance (ANOVA) test, or Mann-Whitney U-test, as appropriate. Correlations were calculated using Pearson correlation analysis. $P<0.05$ was considered statistically significant.

\section{Acknowledgements}

We thank Professor Hongchi Jiang from the First Affiliated Hospital of Harbin Medical University for collecting clinical samples for us.

\footnotetext{
Author details

${ }^{1}$ College of Medical Laboratory Science and Technology, Harbin Medical University (Daqing), 163319 Daqing, P. R. China. ${ }^{2}$ Central Laboratory of Harbin Medical University (Daqing), 163319 Daqing, P. R. China. ${ }^{3}$ Department of Pharmacy, The Second Affiliated Hospital of Chongqing Medical University, 400010 Chongqing, P. R. China. ${ }^{4}$ Department of Pharmacology, Harbin
}

university of commerce, 150081 Harbin, P. R. China. ${ }^{5}$ College of Pharmacy, Shenzhen Technology University, 518118 Shenzhen, P.R. China

\section{Author contributions}

X.Y. conceived the experiments. X.G., J.Z., Y.R., H.P., J.J., Y.Z., X.Z., W.L. and S.S. conducted the experiments. X.G. and Y.R. analysised the data of circRNA expression profiling. S.S. collected and registration of human samples. X.Y. and X.G. analysed and interpreted the data. X.Y. and X.G. wrote the manuscript. X.G. and J.Z. prepared the figures. All authors read and approved the final manuscript. X.G., J.Z. and Y.R. contribute equally for this study and were considered as the co-first author. All authors read and approved the final manuscript.

\section{Funding}

This work was supported by National Natural Science Foundation of China (contract grant numbers: 81800047; 82070053 to X.Y.); Outstanding Youth Fund of Heilongjiang Province (YQ2020H011 to X.Y.); University Nursing Program for Young Scholars with Creative Talents in Heilongjiang Province (contract grant numbers: UNPYSCT-2018067 to X.Y.); Heilongjiang Province Postdoctoral Research Funding (contract grant number: LBH-Q19140 to X.Y.); and National Natural Science Foundation of China (contract grant numbers: 81801484 to Y.R).

\section{Ethics statement}

Tissue specimens were obtained from patients who were diagnosed with HCC between 2017 and 2020 in the Department of The First Affiliated Hospital, Harbin medical university. Every participant involved in this study provided written informed consent that was approved by the Ethics Committee of Harbin medical university (HMUDQ20200010202).

Conflict of interest

The authors declare no competing interests.

\section{Publisher's note}

Springer Nature remains neutral with regard to jurisdictional claims in published maps and institutional affiliations.

Supplementary information The online version contains supplementary material available at https://doi.org/10.1038/s41419-021-03626-7.

Received: 22 November 2020 Revised: 10 March 2021 Accepted: 16 March 2021

Published online: 06 April 2021

\section{References}

1. Craig, A. J., von Felden, J., Garcia-Lezana, T., Sarcognato, S. \& Villanueva, A. Tumour evolution in hepatocellular carcinoma. Nat. Rev. Gastroenterol. Hepatol. 17. 139-152 (2020).

2. Wang, H., Lu, Z. \& Zhao, X. Tumorigenesis, diagnosis, and therapeutic potential of exosomes in liver cancer. J. Hematol. Oncol. 12, 133 (2019).

3. Madkhali, A. A., Fadel, Z. T., Aljiffry, M. M. \& Hassanain, M. M. Surgical treatment for hepatocellular carcinoma. Saudi J. Gastroenterol. 21, 11-17 (2015).

4. Conn, S. J. et al. The RNA binding protein quaking regulates formation of circRNAs. Cell 160, 1125-1134 (2015).

5. Greene, J. et al. Circular RNAs: biogenesis, function and role in human diseases. Front. Mol. Biosci. 4, 38 (2017).

6. Memczak, S. et al. Circular RNAs are a large class of animal RNAs with regulatory potency. Nature 495, 333-338 (2013).

7. Hansen, T. B. et al. Natural RNA circles function as efficient microRNA sponges. Nature 495, 384-388 (2013).

8. $\mathrm{Li}, \mathrm{H}$. et al. circFGFR4 promotes differentiation of myoblasts via binding miR107 to relieve its inhibition of Wnt3a. Mol. Ther. Nucleic Acids 11, 272-283 (2018).

9. Du, W. W. et al. Identifying and characterizing circRNA-protein interaction. Theranostics 7, 4183-4191 (2017).

10. Li, Z. et al. Exon-intron circular RNAs regulate transcription in the nucleus. Nat. Struct. Mol. Biol. 22, 256-264 (2015). 
11. Zlotorynski, E. Non-coding RNA: circular RNAs promote transcription. Nat. Rev. Mol. Cell Biol. 16, 206 (2015).

12. Jeck, W. R. et al. Circular RNAs are abundant, conserved, and associated with ALU repeats. RNA 19, 141-157 (2013).

13. Salzman, J., Chen, R. E., Olsen, M. N., Wang, P. L. \& Brown, P. O. Cell-type specific features of circular RNA expression. PLoS Genet. 9, e1003777 (2013).

14. Zhang, P. F. et al. Circular RNA circTRIM33-12 acts as the sponge of MicroRNA191 to suppress hepatocellular carcinoma progression. Mol. Cancer 18, 105 (2019).

15. Yu, J. et al. Circular RNA cSMARCA5 inhibits growth and metastasis in hepatocellular carcinoma. J. Hepatol. 68, 1214-1227 (2018).

16. Zhu, Y. J. et al. Circular RNAs negatively regulate cancer stem cells by physically binding FMRP against CCAR1 complex in hepatocellular carcinoma. Theranostics 9, 3526-3540 (2019).

17. Wei, Y. et al. A noncoding regulatory RNAs network driven by Circ-CDYL acts specifically in the early stages hepatocellular carcinoma. Hepatology 71, 130-147 (2019).

18. Yu, L. et al. The circular RNA Cdr1as act as an oncogene in hepatocellular carcinoma through targeting miR-7 expression. PLOS ONE 11, e0158347 (2016).

19. Meng, J. et al. Twist1 regulates vimentin through Cul2 circular RNA to promote EMT in hepatocellular carcinoma. Cancer Res. 78, 4150-4162 (2018).

20. Chen, G., Shi, Y., Liu, M. \& Sun, J. circHIPK3 regulates cell proliferation and migration by sponging miR-124 and regulating AQP3 expression in hepatocellular carcinoma. Cell Death Dis. 9, 175 (2018).

21. Shabaninejad, Z. et al. Circular RNAs in cancer: new insights into functions and implications in ovarian cancer. J. Ovarian Res. 12, 84 (2019).

22. Chan, C. C. et al. elF4A3 is a novel component of the exon junction complex. RNA 10, 200-209 (2004).

23. Wang, R. et al. EIF4A3-induced circular RNA MMP9 (circMMP9) acts as a sponge of miR-124 and promotes glioblastoma multiforme cell tumorigenesis. Mol. Cancer 17, 166 (2018)

24. Wei, Y. et al. EIF4A3-induced circular RNA ASAP1(circASAP1) promotes tumorigenesis and temozolomide resistance of glioblastoma via NRAS/MEK1/ ERK1/2 signaling. Preprint at https://doi.org/10.1093/neuonc/noaa214 (2020).

25. Zheng, $X$. et al. The circRNA circSEPT9 mediated by E2F1 and EIF4A3 facilitates the carcinogenesis and development of triple-negative breast cancer. Mol. Cancer 19, 73 (2020).

26. Wang, Y. G. et al. hsa_circ_0091570 acts as a ceRNA to suppress hepatocellular cancer progression by sponging hsa-miR-1307. Cancer Lett. 460, 128-138 (2019).

27. $\mathrm{Xu}$, L. et al. CircSETD3 (Hsa_circ_0000567) acts as a sponge for microRNA-421 inhibiting hepatocellular carcinoma growth. J. Exp. Clin. Cancer Res. 38, 98 (2019).

28. Rossi, F. et al. Circ-ZNF609 regulates G1-S progression in rhabdomyosarcoma. Oncogene 38, 3843-3854 (2019).

29. Wu, J. et al. CirclRAK3 sponges miR-3607 to facilitate breast cancer metastasis Cancer Lett. 430, 179-192 (2018).

30. Chen, Z. et al. Hsa circ 101555 functions as a competing endogenous RNA of miR-597-5p to promote colorectal cancer progression. Oncogene $\mathbf{3 8}$ 6017-6034 (2019).

31. Wang, Y. et al. Chemotherapy-induced miRNA-29c/catenin-delta signaling suppresses metastasis in gastric cancer. Cancer Res. 75, 1332-1344 (2015).

32. Liang, $Y$. et al. Targeting the circBMPR2/miR-553/USP4 axis as a potent therapeutic approach for breast cancer. Mol. Ther. Nucleic Acids 17, 347-361 (2019).

33. $\mathrm{Yu}$, J. et al. CircRNA-104718 acts as competing endogenous RNA and promotes hepatocellular carcinoma progression through microRNA-218-5p/ TXNDC5 signaling pathway. Clin. Sci. 133, 1487-1503 (2019).
34. Chen, $X$. et al. Circular RNA circHIPK3 modulates autophagy via MIR124-3pSTAT3-PRKAA/AMPKalpha signaling in STK11 mutant lung cancer. Autophagy 16, 659-671 (2019).

35. Lu, Q. et al. Circular RNA circSLC8A1 acts as a sponge of miR-130b/miR-494 in suppressing bladder cancer progression via regulating PTEN. Mol. Cancer 18, 111 (2019).

36. Zheng, $X$. et al. A novel protein encoded by a circular RNA circPPP1R12A promotes tumor pathogenesis and metastasis of colon cancer via Hippo-YAP signaling. Mol. Cancer 18, 47 (2019).

37. Wang, M., Yu, F. \& Li, P. Circular RNAs: characteristics, function and clinical significance in hepatocellular carcinoma. Cancers 10, e258 (2018).

38. Hu, Z. Q. et al. Circular RNA sequencing identifies CircASAP1 as a key regulator in hepatocellular carcinoma metastasis. Hepatology 72, 906-922 (2019).

39. Turato, C. et al. MiR-122 targets serpinB3 and is involved in sorafenib resistance in hepatocellular carcinoma. J. Clin. Med. 8, e171 (2019).

40. Xie, Y. et al. MiR-6875-3p promotes the proliferation, invasion and metastasis of hepatocellular carcinoma via BTG2/FAKAkt pathway. J. Exp. Clin. Cancer Res. 38, 7 (2019).

41. Zhou, Y. et al. Hepatocellular carcinoma-derived exosomal miRNA-21 contributes to tumor progression by converting hepatocyte stellate cells to cancer-associated fibroblasts. J. Exp. Clin. Cancer Res. 37, 324 (2018).

42. Shi, D. M. et al. miR-296-5p suppresses EMT of hepatocellular carcinoma via attenuating NRG1/ERBB2/ERBB3 signaling. J. Exp. Clin. Cancer Res. 37, 294 (2018).

43. Mirzaei, H. R. et al. Circulating microRNAs in hepatocellular carcinoma: potential diagnostic and prognostic biomarkers. Curr. Pharm. Des. 22, 5257-5269 (2016).

44. Chen, J. et al. circPTN sponges miR-145-5p/miR-330-5p to promote proliferation and stemness in glioma. J. Exp. Clin. Cancer Res. 38, 398 (2019).

45. Hsu, W. C. et al. MicroRNA-145 suppresses cell migration and invasion in upper tract urothelial carcinoma by targeting ARF6. FASEB J. 34, 5975-5992 (2020).

46. Zhang, Z. et al. CircDUSP16 promotes the tumorigenesis and invasion of gastric cancer by sponging miR-145-5p. Gastric Cancer 23, 437-448 (2020).

47. Ashwal-Fluss, R. et al. circRNA biogenesis competes with pre-mRNA splicing. Mol. Cell 56, 55-66 (2014).

48. Zhang, $Y$. et al. CDCA3 is a potential prognostic marker that promotes cell proliferation in gastric cancer. Oncol. Rep. 41, 2471-2481 (2019).

49. Qian, W. et al. CDCA3 mediates p21-dependent proliferation by regulating E2F1 expression in colorectal cancer. Int. J. Oncol. 53, 2021-2033 (2018).

50. Adams, M. N. et al. Expression of CDCA3 is a prognostic biomarker and potential therapeutic target in non-small cell lung cancer. J. Thorac. Oncol. 12 1071-1084 (2017).

51. Chen, Q., Zhou, L., Ye, X., Tao, M. \& Wu, J. miR-145-5p suppresses proliferation, metastasis and EMT of colorectal cancer by targeting CDCA3. Pathol. Res. Pract. 216, e152872 (2020)

52. Beermann, J., Piccoli, M. T., Viereck, J. \& Thum, T. Non-coding RNAs in development and disease: background, mechanisms, and therapeutic approaches. Physiol. Rev. 96, 1297-1325 (2016).

53. Lin, Y. et al. Systematic analysis of gene expression alteration and coexpression network of eukaryotic initiation factor 4A-3 in cancer. J. Cancer 9 4568-4577 (2018).

54. Legnini, I. et al. Circ-ZNF609 is a circular RNA that can be translated and functions in myogenesis. Mol. Cell 66, 22-37 (2017). e29.

55. Glazar, P., Papavasileiou, P. \& Rajewsky, N. circBase: a database for circular RNAs RNA 20, 1666-1670 (2014). 